\title{
High-Integrity IMM-EKF-Based Road Vehicle Navigation With Low-Cost GPS/SBAS/INS
}

\author{
Rafael Toledo-Moreo, Miguel A. Zamora-Izquierdo, Benito Úbeda-Miñarro, \\ and Antonio F. Gómez-Skarmeta
}

\begin{abstract}
User requirements for the performance of Global Navigation Satellite System (GNSS)-based road applications have been significantly increasing in recent years. Safety systems based on vehicle localization, electronic fee-collection systems, and traveler information services are just a few examples of interesting applications requiring onboard equipment (OBE) capable of offering a high available accurate position, even in unfriendly environments with low satellite visibility such as built-up areas or tunnels and at low cost. In addition to that, users and service providers demand from the OBEs not only accurate continuous positioning but integrity information of the reliability of this position as well. Specifically, in life-critical applications, high-integrity monitored positioning is absolutely required. This paper presents a solution based on the fusion of GNSS and inertial sensors (a Global Positioning System/Satellite-Based Augmentation System/Inertial Navigation System integrated system) running an extended Kalman filter combined with an interactive multimodel method (IMM-EKF). The solution developed in this paper supplies continuous positioning in marketable conditions and a meaningful trust level of the given solution. A set of tests performed in controlled and real scenarios proves the suitability of the proposed IMM-EKF implementation as compared with lowcost GNSS-based solutions, dead reckoning systems, single-model EKF, and other filtering approaches of the current literature.
\end{abstract}

Index Terms -Data fusion, inertial sensors, integrity, interactive multimodel (IMM), navigation systems, satellite navigation.

\section{INTRODUCTION}

$\mathbf{R}$ OAD vehicle navigation systems are one of the main fields of interest in the world of Intelligent Transport Systems. Road applications such as traveler information, route guidance, automatic emergency calls, freight management, advanced driver assistance, or electronic fee collection require an onboard equipment (OBE) capable of offering high available accurate position at a low price [1].

For this purpose, much current research focuses on the combination of odometry, low-cost inertial units, and satellite navigation [2]-[10]. The approach of fusing GPS with

Manuscript received April 28, 2006; revised November 3, 2006, January 22, 2007, and March 16, 2007. This work was supported in part by the Spanish Ministerio de Fomento under Grant FOM/3929/2005 and by the Galileo Joint Undertaking (GJU) under Grant GIROADS 332599. The Associate Editor for this paper was Y. Wang.

The authors are with the Department of Information and Communication Engineering, Computer Science Faculty, University of Murcia, 30071 Murcia, Spain (e-mail: toledo@um.es; mzamora@um.es; bubeda@um.es; skarmeta@dif.um.es)

Color versions of one or more of the figures in this paper are available online at http://ieeexplore.ieee.org.

Digital Object Identifier 10.1109/TITS.2007.902642 odometry information [coming from either the Anti-Blocking System (ABS) system or odometers installed in the vehicle], as developed in [2] and [3], presents the main disadvantage of the lack of precision in the heading. To increase the accuracy of the heading estimate, some authors propose a combination of gyro, odometers, and GPS receivers [4]-[6]. More precise systems such as those in [7] and [8] employ GPS/Inertial Navigation System (INS) at the expense of higher costs. However, further investigations must be performed to maintain the quality of the performance of these systems at lower costs.

In this paper, the navigation system is integrated by SatelliteBased Augmentation System (SBAS)-capable GPS sensors, low-cost inertial accelerometers and gyros, and the odometry information coming from the ABS system of the vehicle. The use of SBAS-capable GPS sensors provides higher levels of performance in satellite positioning and useful integrity parameters, such as the Horizontal Protection Level HPL SBAS $_{3}$ (a.k.a. HPL WAAS) parameter [11]. Note that in order to employ inertial units in mass-market applications, only low-cost inertial units based on microelectromechanical (MEM) technology are affordable [12]. MEM-based inertial sensors are much cheaper than other inertial sensors based on traditional technologies such as the ring laser or fiber optic gyros, but they offer much lower levels of performance. On the other hand, the use of the odometry information coming from the ABS system of the vehicle supplies low-quality velocity information without any additional cost.

To fuse the information coming from the sensors, different approaches can be found in the actual literature. Many of them rely on the implementation of an extended Kalman filter (EKF) [2], [3], [5], [6], [9], [13]. The performance of the EKF is reliable in many practical situations, but the nonlinear state equations may lead to instability problems. Other filtering methods can be found in the literature, such as the unscented Kalman filter (UKF) and the particle-based solution [2], [14], [34]. Of special interest in the implementation of the filter is the selection of the proper model for the road vehicle. Many authors consider similar models to those applied to indoor robots [15]-[17]. In most of the cases, the vector state of the model is defined as the vehicle pose (position and orientation) in Cartesian coordinates, and vehicle movement is considered as consecutive constant rate curves around their instant rotation centers. Actual interesting kinematic models can be found in [4], [5], and [8]. These models offer good solutions under certain movement restrictions. However, the problem of a model capable to represent vehicle movements along roads in usual 
driving situations independent of the maneuvering state, traffic conditions, etc., is still under intensive research.

This paper presents an interactive multimodel (IMM)-based method applied to the fusion filtering of inertial, odometry, and satellite data. The implementation of IMM-based methods allows the possibility of using highly dynamic models just when required, diminishing unrealistic noise considerations in nonmaneuvering situations, and the computational charge of the system. The idea of defining different models according to the situation in which the vehicle is involved is not new, and it has been applied to the aerial navigation field in the last times [18], [19]. Lately, some authors have considered its application in the navigation systems of road vehicles. In [20], an IMM method is applied to the problem of object tracking with a video system in a car. In [21], an extended IMM implementation is used for car tracking from a range image sequence. The authors in [22] develop an IMM algorithm to detect lane change maneuvers based on laser, radar, and vision data.

In our approach, the use of an IMM method allows exploiting the benefits of high dynamic models in the problem of road vehicle positioning while avoiding their disadvantages regarding computational charge and unrealistic noise considerations. Two models of the vehicle have been defined to reproduce its movements along roads. The straight (or nonmaneuvering) model reproduces properly straight and mild trajectories of the vehicle. The curved (or maneuvering) model considers sharp turns and brusque accelerations in the vehicle state at the expense of higher noise considerations. The IMM-EKF filter that was developed calculates the probability of success of each model at every filter execution scan, supplying a realistic combined solution for vehicle behavior. These probabilities are calculated according to a Markov model for the transition between maneuver states.

The integrity concept in navigation systems can be defined as the capability of the system to detect anomalies and warn the user when the system should not be used. Furthermore, a high-integrity navigation system must be able to reject fake measurements from the solution and provide an integrity parameter of the confidence on the system performance anytime. In many interesting applications such as GPS-based electronic feecollection systems, this capacity to perform a self-evaluation and provide an uninterrupted meaningful integrity parameter is crucial [23].

Traditional GPS receivers provide precision parameters based on Geometry Dilution of Precision calculations. Various parameters are based on this concept, i.e., Position Dilution of Precision, Time Dilution of Precision, Horizontal Dilution of Precision (HDOP), and Vertical Dilution of Precision. The algorithms used to calculate these values can be found in [24]. All of them are exclusively based on the geometry of the satellite constellation used in the GPS solution, and errors caused by wrong pseudorange measurements are not considered.

Currently, two different approaches to provide integrity in GPS-based navigation systems are Receiver Autonomous Integrity Monitoring (RAIM) and the use of SBAS. The RAIM technique, which is based on an overdetermined solution to evaluate its consistency, requires a minimum of five satellites to detect a satellite anomaly and six or more to be able to reject it [24]. Unfortunately, this cannot be assumed in usual traffic situations, especially in cities. The use of SBAS allows one to have integrity information through the geostationary satellite. SBASs offer to their client equipments the possibility of calculating an indicative value of position integrity that considers pseudorange errors, i.e., HPL $L_{\text {SBAS }}$. These messages can be obtained in Europe via the geostationary satellite by using the European Geostationary Navigation Overlay System (EGNOS) or via the Internet through Signal In Space through Internet (SISNeT). However, despite the improvements achieved, the complete dependency on GPS coverage and the fact that this integrity information is only based on Global Navigation Satellite System (GNSS) measurements encourage the definition of a continuous integrity parameter capable of meaningfully modeling the positioning quality of the whole multisensor navigation system.

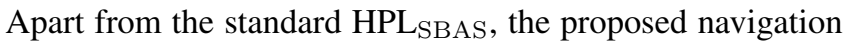
system continuously provides the Horizontal Trust Level (HTL) parameter [1], i.e., a meaningful value of confidence on the positioning, which depends on the sensor variances and the state and covariance of the multisensor data filter anytime.

The rest of this paper is organized as follows: First, a description of the proposed navigation system is presented, including EKF, UKF, and IMM-EKF filters, and a description of the hardware in the OBE. Next, results achieved in the tests are analyzed and discussed. Finally, the conclusions obtained from this paper are commented.

\section{SySTEM DESCRIPTION}

The solution presented in this paper is based on a GPS/SBAS/INS-integrated system. Next, a brief introduction to the European SBAS systems (EGNOS and SISNeT) and the INS units is done.

\section{A. European SBAS Systems: EGNOS and SISNeT}

The use of combined GPS/SBAS systems, as compared to a single GPS solution, provides noticeable improvements. EGNOS users access corrected GPS positions, along with integrity information, allowing the calculation of HPL and Vertical Protection Level parameters. In [11], the results of several static trials with and without EGNOS values are shown. According to the authors, EGNOS can decrease the horizontal position error (HPE95) values from 3.22 to $1.38 \mathrm{~m}$ when no masking was applied (the HPE value can be interpreted as the maximum position error that can occur and go undetected). In similar conditions but applying mask values of $30^{\circ}$, HPE95 values decrease from 6.85 to $2.23 \mathrm{~m}$. In the vertical axis, the VPE95 value diminishes from 18.72 to $0.53 \mathrm{~m}$ when $30^{\circ}$ masking was applied. SISNeT supplies the same corrections through the Internet in order to avoid the visibility problems of the geostationary satellite.

Despite these improvements, GPS/SBAS systems cannot fulfill the requirements of high-integrity demanding applications, especially in city environments, mainly due to the poor GPS coverage in built-up areas. 


\section{B. INSs}

Inertial Measurement Units (IMUs) supply accelerations and rates of turn relative to the three Cartesian axes of the sensor body frame. Although these measurements complement the GPS/SBAS lacks and provide positioning during the outages of the satellite signal, the double integration process required to obtain position from the acceleration measurements is the main source of error for the INS units. In order to avoid excessive drifts, often updates must be performed by a global system. In addition, only low-cost inertial units based on MEM technology are affordable considering a real mass-market OBE. Unfortunately, these sensors present bad noise features and drifts, and the implementation of error models is advisable. In order to diminish the drifts during the GPS outages, odometry measurements coming from the ABS encoders of the vehicle are also considered in our system. The ABS system provides nonprecise velocity information with a very low increase of the final cost, since no further installations or sensors are needed. Apart from the precision problem due to the low level of performance of the ABS encoders, typical odometry problems such as glides, unequal wheel diameters, or effective wheel diameter uncertainty are also presented.

To obtain the proper inputs to the data fusion filter from the raw measurements coming from the sensors, observation models are implemented, and considerations about sensor performances are done.

\section{Multisensor Data Fusion Filter}

The data fusion filter that was developed to combine information coming from GPS/SBAS, INS, and odometry sensors is based on a loosely coupled EKF architecture implementing an IMM method to employ the vehicle model definition that better describes the current vehicle behavior.

1) Kalman Filter: The Kalman filter is a recursive leastsquares estimator [26], [27]. It produces at time $k$ a minimum mean-squared error estimate $\hat{\mathbf{x}}(k \mid k)$ of a state vector $\mathbf{x}(k)$. This estimate is obtained by fusing a state estimate prediction $\hat{\mathbf{x}}(k \mid k-1)$ with an observation $\mathbf{z}(k)$ of the state vector $\mathbf{x}(k)$. The estimate $\hat{\mathbf{x}}(k \mid k)$ is the conditional mean of $\mathbf{x}(k)$ given all observations $\mathbf{Z}^{k}=[\mathbf{z}(1), \ldots, \mathbf{z}(k)]$ up until time $k$, i.e.,

$$
\hat{\mathbf{x}}(k \mid k)=\mathbf{E}\left[\mathbf{x} \mid \mathbf{Z}^{k}\right]
$$

where $\mathbf{Z}^{k}$ is the sequence of all observations up until time $k$.

2) Vehicle Models: In order to represent the movements of the vehicle along roads, two models have been developed. Both are based on the rigid solid definition of a four-wheel vehicle, the back wheels of which can rotate only about a transversal axis of the vehicle, and the forward wheels turn describing curves centered in their instant rotation center. Fig. 1 graphically shows the kinematical model and its nomenclature, which is based on [28]. In the proposed model, $x_{c}(k), y_{c}(k)$ are the coordinates of the geometrical center of the vehicle (g.c.), $\theta(k)$ is the vehicle orientation, $v_{c}(k)$ is the velocity in g.c., $\phi_{c}(k)$ is the angle of the velocity $v_{c}(k)$, and $s_{c}(k)$ is the slide correction angle. The $s_{c}(k)$ term represents the slip bias angular component in g.c. that effectively causes the

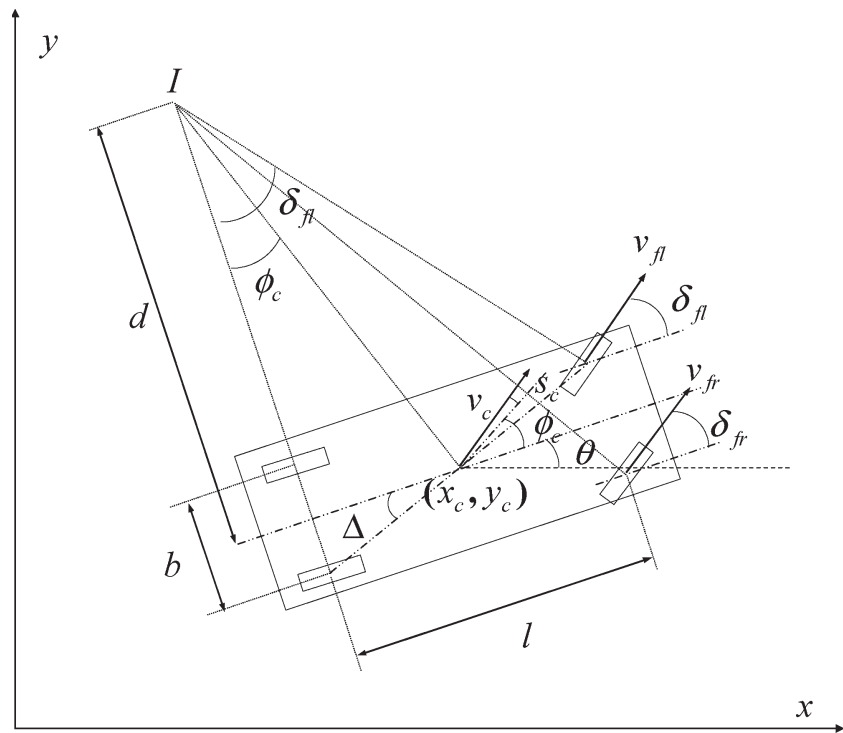

Fig. 1. Kinematical model.

vehicle to deviate from its ideal course, which is typically a consequence of unbalanced weight distribution and inaccurate wheel alignment. To represent this, sideslip angle variations could also be taken into account, and they are being considered for future investigations.

Thus, the final velocity angle referred to the North is given by the addition of $\theta(k), \phi_{c}(k)$, and $s_{c}(k) . v_{\mathrm{ff}}, \delta_{\mathrm{f}}$ and $v_{\mathrm{fr}}, \delta_{\mathrm{fr}}$ are the velocities and angles of the forward left and right wheels, respectively. $I$ represents the instant center of rotation, and $b, l$, and $\Delta$ define the vehicle geometry.

The straight model or nonmaneuvering model represents a basic nonmaneuvering behavior of the vehicle with its transition equation defined as

$$
\begin{aligned}
x_{c}(k+1)= & x_{c}(k)+T v_{c}(k) \cos \left(\theta(k)+\phi_{c}(k)+s_{c}(k)\right) \\
& +0.5 T^{2} \dot{v}_{c}(k) \cos \left(\theta(k)+\phi_{c}(k)+s_{c}(k)\right) \\
& -0.5 T^{2} v_{c}(k) \dot{\theta}(k) \sin \left(\theta(k)+\phi_{c}(k)+s_{c}(k)\right) \\
y_{c}(k+1)= & y_{c}(k)+T v_{c}(k) \sin \left(\theta(k)+\phi_{c}(k)+s_{c}(k)\right) \\
& +0.5 T^{2} \dot{v}_{c}(k) \sin \left(\theta(k)+\phi_{c}(k)+s_{c}(k)\right) \\
& +0.5 T^{2} v_{c}(k) \dot{\theta}(k) \cos \left(\theta(k)+\phi_{c}(k)+s_{c}(k)\right) \\
\theta(k+1)= & \theta(k)+T \dot{\theta}(k)+0.5 T^{2} \ddot{\theta}(k) \\
\dot{\theta}(k+1)= & \dot{\theta}(k)+T \ddot{\theta}(k) \\
v_{c}(k+1)= & v_{c}(k)+T \dot{v}_{c}(k) \\
\phi_{c}(k+1)= & \phi_{c}(k)+T \dot{\phi}_{c}(k) \\
s_{c}(k+1)= & s_{c}(k)+T \dot{s}_{c}(k)
\end{aligned}
$$

as noted by

$$
\mathbf{x}(k+1)=f(\mathbf{x}(k))+G(\mathbf{x}(k)) v(k)
$$


where $f$ is the state transition matrix, $G$ is the noise matrix, and the state and noise vectors are

$$
\begin{aligned}
& \mathbf{x}(k)=\left[x_{c}(k) y_{c}(k) \theta(k) \dot{\theta}(k) v_{c}(k) \phi_{c}(k) s_{c}(k)\right]^{T} \\
& v(k)=\left[\ddot{\theta}(k) \dot{v}_{c}(k) \dot{\phi}_{c}(k) \dot{s}_{c}(k)\right]^{T} .
\end{aligned}
$$

In the straight model, $\phi_{c}(k)$ is modeled by a first-order function. Hence, both straight and mild trajectories fulfill the kinematical definition of the model. However, when sharp curves are performed, it is advisable to represent $\phi_{c}(k)$ by a second-order equation. Thus, the state and noise vectors of the curved model (or maneuvering model) are

$$
\begin{aligned}
& \mathbf{x}(k)=\left[x_{c}(k) y_{c}(k) \theta(k) \dot{\theta}(k) v_{c}(k) \phi_{c}(k) \dot{\phi}_{c}(k) s_{c}(k)\right]^{T} \\
& v(k)=\left[\ddot{\theta}(k) \dot{v}_{c}(k) \ddot{\phi}_{c}(k) \dot{s}_{c}(k)\right]^{T}
\end{aligned}
$$

and the transition equation for this model can be easily obtained analogous to (1).

3) Sensor Models: In order to obtain the filter observations $\mathbf{z}(k)$ at scan $k$, different transformations must be done. The observation vector of our system is defined as

$$
\begin{aligned}
\mathbf{z}(k)=\left[x_{c}^{G}(k) y_{c}^{G}(k) x_{c}^{I}(k)\right. & y_{c}^{I}(k) \\
& \left.\theta^{I}(k) \dot{\theta}^{O}(k) v_{c}^{O}(k) \phi_{c}^{O}(k) v_{c}^{I}(k)\right]^{T}
\end{aligned}
$$

where $x_{c}^{G}(k), y_{c}^{G}(k)$ and $x_{c}^{I}(k), y_{c}^{I}(k)$ are the Cartesian coordinates of the g.c. according to the GPS/SBAS and INS measurements, respectively, $\theta^{I}(k)$ and $v_{c}^{I}(k)$, which are obtained from inertial measurements, define severally the orientation and velocity of the g.c. of the vehicle, and finally, $\dot{\theta}^{O}(k)$, $v_{c}^{O}(k)$, and $\phi_{c}^{O}(k)$ are, respectively, the angular velocity, linear velocity, and its angle in the g.c. observed by the odometry system. In this section, we present the transformations required to obtain the observations $\dot{\theta}^{O}(k), v_{c}^{O}(k), \phi_{c}^{O}(k), x_{c}^{I}(k), y_{c}^{I}(k)$, and $v_{c}^{I}(k)$. The rest of the observation variables can be easily obtained from the sensor measurements.

4) Odometry Observations: Considering the vehicle as a rigid solid and the assumptions done in [28], the velocity in the g.c. $v_{c}(k)$ can be calculated as

$$
v_{c}(k)^{O}=v_{\mathrm{fl}}(k) \frac{\cos \left(\Delta-\delta_{\mathrm{fl}}(k)\right)}{\cos \left(\Delta-\phi_{c}(k)-s_{c}(k)\right)}
$$

where $v_{\mathrm{fl}}(k)$ and $\delta_{\mathrm{fl}}(k)$ are, respectively, the velocity and angle of the forward left wheel. The use of the front left wheel data (or the front right wheel), as compared to the measurements coming from the rear wheels, has the benefit of allowing the calculation of the angular velocity, which can be calculated by applying (3) if it was assumed that the velocity projection in the g.c. equals the wheel velocity projection over the diagonal

$$
\dot{\theta}^{O}(k)=v_{\mathrm{fl}}(k) \frac{\sin \left(\delta_{\mathrm{fl}}(k)\right)}{l} .
$$

Finally, to calculate the angle of the velocity in the g.c. (Fig. 1), the geometrical transformations from the angles of the forward wheels to the g.c. are given by

$$
\begin{aligned}
& d=\frac{l}{\tan \left(\delta_{\mathrm{fl}}\right)}+\frac{b}{2} \\
& \tan \left(\phi_{c}(k)+s_{c}(k)\right)=\frac{l / 2}{d} .
\end{aligned}
$$

Thus, the angle of the velocity is

$$
\phi_{c}^{O}(k)=\arctan \left(\frac{l \cdot \tan \left(\delta_{\mathrm{fl}}(k)\right)}{2 l+b \cdot \tan \left(\delta_{\mathrm{fl}}(k)\right)}\right)-s_{c}(k) .
$$

5) Inertial Observations: To obtain the inertial observations, four different phases must be performed.

First, whereas low-cost inertial sensors are used, error models must be considered. Hence, the first step must be the implementation of error models for the inertial measurements. The models implemented in this paper are based on the work by Barshan and Durrant-Whyte [29] and can be described by

$$
\varepsilon=C_{1}\left(1-e^{\frac{-t}{\tau}}\right)+C_{2}
$$

where $\varepsilon$ represents the error model for the acceleration in the body frame of the sensor, and $C_{1}, C_{2}$ (in meters per second square), and $\tau$ (in seconds) are model parameters. Fig. 2 shows the values of the forward acceleration measurement during the compensation test and the outcome model. During this test, no forces were applied to the sensors (except for the Earth's gravity), and no external updates were performed. In order to avoid gravity component interferences, the IMU was stabilized by using calibrated gyros and tilts [30].

The adjustment of the model parameters was performed by using a Nelder-Mead nonrestricted nonlinear multidimensional method, where the minimizing function was the mean squared error [31]. In the test shown in Fig. 2, the parameter values obtained were $C_{1}=-0.0043, C_{2}=-0.007$, and $\tau=500$, which result in a mean value of $-3.2172 \times 10^{-4}$ and a standard deviation of 0.0033 . With these values, the position drifted $70 \mathrm{~cm}$ after $60 \mathrm{~s}$. In the same test but without applying any error model, the position drifted up to $55 \mathrm{~m}$.

Second, in order to obtain the acceleration vector referred to the global frame (North-East-Down) (G) from the local reference $(\mathbf{S})$, we can use the rotation matrix shown at the bottom of the next page, where $\psi$ is the yaw, $\theta$ is the pitch, and $\phi$ is the roll in the body frame $\mathbf{S}$.

Then, a gravitational model must be applied to compensate the Earth gravity effects. Typically, in terrestrial applications with mobile units, the gravity is assumed to be $-9.81 \mathrm{~m} / \mathrm{s}^{2}$ in the $z$-axis and being zero in the two other components in the global reference frame (local tangent plane).

As a final step, the inertial observations $x_{c}^{I}$ and $y_{c}^{I}$ can be calculated by applying

$$
\begin{aligned}
& x_{c}^{I}(k+1)=x_{c}(k)+v_{c_{x}}(k) T+0.5 \cdot a_{x} T^{2} \\
& y_{c}^{I}(k+1)=y_{c}(k)+v_{c_{y}}(k) T+0.5 \cdot a_{y} T^{2}
\end{aligned}
$$




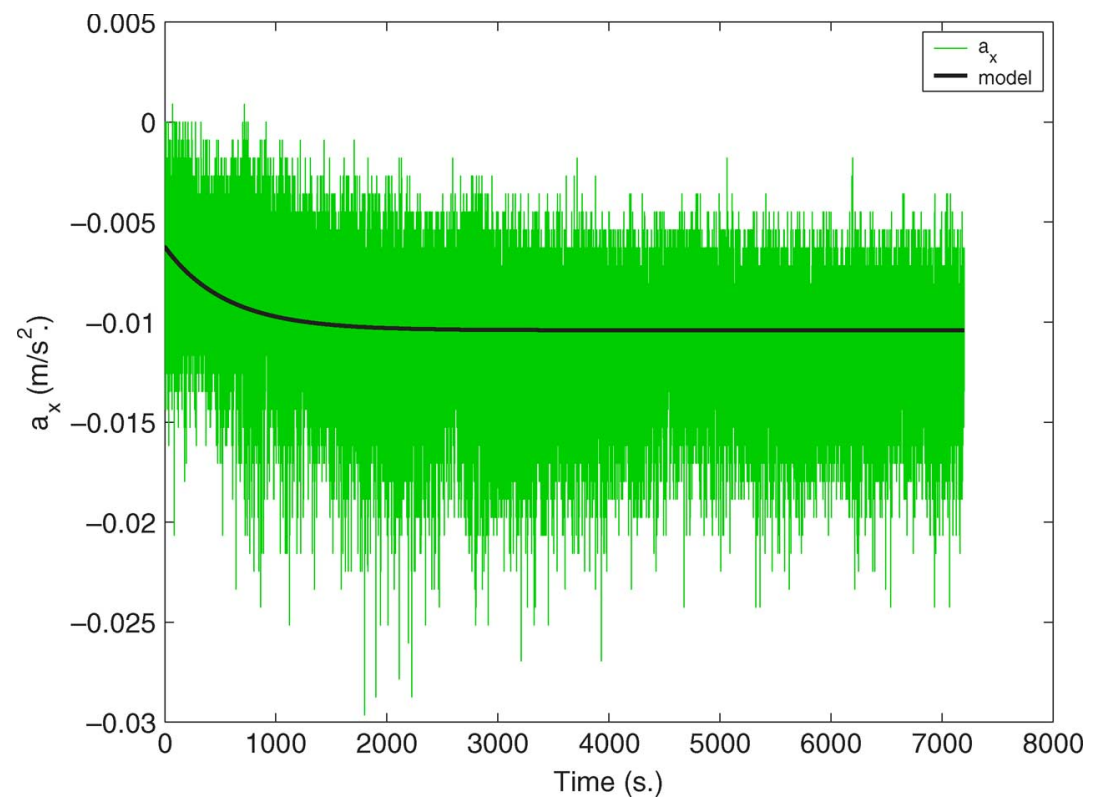

Fig. 2. Forward acceleration measurements and the model obtained in the error model test.

where $x_{c}(k)$ and $y_{c}(k)$ are the state variables just after the last update, $T$ is the difference between the time stamp of the inertial measurements and the time stamp of the last measurement that updated the state vector, $a_{x}$ and $a_{y}$ are the acceleration values in the global reference system, as obtained from the previous step, and the values of velocities $v_{c_{x}}$ and $v_{c_{y}}$ are given by

$$
\begin{aligned}
& v_{c_{x}}(k)=v_{c}(k) \cos \left(\theta(k)+\phi_{c}(k)+s_{c}(k)\right) \\
& v_{c_{y}}(k)=v_{c}(k) \sin \left(\theta(k)+\phi_{c}(k)+s_{c}(k)\right) .
\end{aligned}
$$

The observation $v_{c}^{I}$ can be calculated by using

$$
v_{c}^{I}(k+1)=v_{c}(k)+a_{t}^{I} T
$$

where $v_{c}(k)$ is the state variable just after the last update, and $a_{t}^{I}$ represents the module of the acceleration tangential to the vehicle trajectory, which is calculated according to the inertial measurements. To calculate $a_{t}^{I}$, we will assume that the geometrical and gravity centers of the vehicle coincide in $\left(x_{c}, y_{c}\right)$. Naming $\alpha$ as the angle between the absolute acceleration vector of the vehicle $\mathbf{a}$ and the $x$-axis, we can affirm that

$$
\alpha=\arccos \left(\frac{a_{x}}{a}\right), \quad a=\sqrt{a_{x}^{2}+a_{y}^{2}}
$$

where $a_{x}$ and $a_{y}$ are the horizontal components of the vector a and $a$ its projection on the $x y$ plane. Besides, the module of the tangential acceleration can be calculated as

$$
a_{t}^{I}=a \cos \left(\alpha-\left(\theta+\phi_{c}+s_{c}\right)\right)
$$

Thus, the next expression for the $a_{t}^{I}$ value can be obtained as

$$
\begin{aligned}
a_{t}^{I}=\sqrt{a_{x}^{2}+a_{y}^{2}} \cdot \cos (\arccos ( & \left.\frac{a_{x}}{a}\right) \\
& \left.-\left(\theta(k)+\phi_{c}(k)+s_{c}(k)\right)\right) .
\end{aligned}
$$

6) Error Considerations: In the observation models proposed for the odometry and inertial sensors, the observation model is a function of the state of the vehicle. This could lead to some undesirable filter inconsistencies, although it can also bring some benefits to filter implementation. In order to accommodate the complexity of adding nonwhite noise to the observations, which violates the noncorrelation hypothesis of the Kalman filter, we considered the augmentation of the state vector to estimate this correlated noise during the estimation process. However, in the correlation tests performed to analyze this, the autocorrelation function was not found significant, and the low biases and appropriate performance in the results discouraged this approach. This problem has already been considered in the current literature. Several authors such as Tham et al. [28] or Guivant et al. [37] define the observation models as a function of the vehicle state in order to transform the odometry measurements of the wheels into their g.c. projections by taking

$$
{ }^{G S} R=\left[\begin{array}{ccc}
\cos \theta \cos \psi & \sin \phi \sin \theta \cos \psi-\cos \phi \sin \psi & \cos \phi \sin \theta \cos \psi+\sin \phi \sin \psi \\
\cos \theta \sin \psi & \sin \phi \sin \theta \sin \psi+\cos \phi \cos \psi & \cos \phi \sin \theta \sin \psi-\sin \phi \cos \psi \\
-\sin \theta & \sin \phi \cos \theta & \cos \phi \cos \theta
\end{array}\right]
$$


this into account in the consideration of sensor error estimation. Similar considerations as those presented in these papers have been done in the estimation of odometry observation errors.

For accelerometer measurements, the proposed error estimation is based on the work by Sukkarieh et al. [38]. Two different observation faults are distinguished: 1) low-frequency faults due to sensor biases and the IMU misalignment and 2) high-frequency faults due to multipath errors in the GPS observations. High-frequency faults are detected by means of the Normalized Innovation Squared (NIS) inequation, as explained in Section II-D2. For the low-frequency faults, noise terms have to be considered in the filter. The expression

$$
a_{i}=a_{i T}+b_{a_{i}}+\nu
$$

describes the error consideration in the acceleration observations, after the correction process explained in Section II-C5, which was used for the error considerations in the observation covariance matrix, where $a_{i}$ is the acceleration observation, $a_{i T}$ is the real observation, $b_{a_{i}}$ is defined as the acceleration bias, and $\nu$ represents the white noise.

An estimation of the errors committed due to the application of (4) and (6) to the obtained velocity and position can be defined by the following expressions, which are analogous to $(5)$ :

$$
\begin{aligned}
& v_{i}=v_{i T}+b_{a_{i}} t+\int \nu d t+\eta_{v} \\
& x_{i}=x_{i T}+\frac{b_{a_{i}} t^{2}}{2}+\iint \nu d t+\eta_{x}
\end{aligned}
$$

where $\int \nu$ represents a random walk due to unconsidered errors, and $\eta_{v}$ and $\eta_{x}$ describe the velocity and position error terms due to the variances of the corresponding $v_{c}(k)$ and $x_{c}(k)$ variables of the previous state given by the state covariance matrix $P(k)$.

The proposed approach has the benefits of deflecting nonlinearities and state dependency in the $H$ matrix of the filter, which avoids the undesirable consequences of the Jacobian approximation and its calculation referred to the predicted state, at the expense of new error considerations in the observation variables.

\section{EKF Implementation}

The execution of the EKF can be divided into the following three phases.

1) Prediction and Observation: We have already seen the kinematical models of the vehicle. These models are now used to perform state prediction. Taking into account (2), the equation for state prediction is

$$
\hat{\mathbf{x}}(k+1 \mid k)=f(\hat{\mathbf{x}}(k \mid k))
$$

where $f$ represents the transition matrix. The covariance of the state prediction $P(k+1 \mid k)$ is calculated as

$$
P(k+1 \mid k)=F_{x}(k) P(k \mid k) F_{x}^{T}(k)+G(k) Q(k) G(k)^{T}
$$

where $G(k)$ is the gain matrix multiplying the noise vector $v(k)$. Finally, $F_{x}(k)$ is the Jacobian of the transition matrix regarding the state

$$
F_{x}(k)=\left.\frac{\partial f(\mathbf{x}(k))}{\partial \mathbf{x}(k)}\right|_{\mathbf{x}=\hat{\mathbf{x}}(k \mid k)}
$$

and $Q(k)$ is the covariance noise matrix of the noise vector $v(k)$. The observation model is described by

$$
\mathbf{z}(k)=H \cdot \mathbf{x}(k)+\omega(k), \quad \omega(K) \sim N(0, R(k)) .
$$

The relation between vector state and vector observation is linear and constant (matrix $H$ ), and the equation can be expressed as

$$
\hat{\mathbf{z}}(k+1)=H \cdot \hat{\mathbf{x}}(k+1 \mid k)
$$

2) Validation: After obtaining the observation vector $\mathbf{z}(k+1)$, the innovation vector can be calculated as

$$
\nu(k+1)=\mathbf{z}(k+1)-\hat{\mathbf{z}}(k+1) .
$$

The validation region is calculated following a $\chi^{2}$ distribution like

$$
\nu^{T}(k+1) S^{-1}(k+1) \nu(k+1) \leq g^{2} .
$$

This expression represents an ellipsoid of probability called NIS, where the threshold $g$ is the number of sigmas in the region [29]. Since the innovation vector is dynamically created according the measurements available at every scan, fault detection is realized for every sensor vector, which facilitates the spurious detection. The innovation covariance is

$$
S(k+1)=H P(k+1 \mid k) H^{T}+R(k+1) .
$$

3) Update: Once the innovation covariance $S$ is known, the Kalman gain can be calculated as

$$
W(k+1)=P(k+1 \mid k) H^{T} S^{-1}(k+1) .
$$

The estimate position of the vehicle supplied by the filter is

$$
\hat{\mathbf{x}}(k+1 \mid k+1)=\hat{x}(k+1 \mid k)+W(k+1) \nu(k+1)
$$

and the updated covariance is

$$
P(k+1 \mid k+1)=P(k+1 \mid k)-W(k+1) S(k+1) W^{T}(k+1) .
$$

Detailed development of the EKF equations for both maneuvering and nonmaneuvering models can be found in [30].

\section{E. $U K F$}

As mentioned in Section I, some other methods apart from EKF have been implemented to deal with the Kalman filter linearity assumptions. In the current literature, several examples of the improvements of the UKF approach as compared to EKF can be found for problem solving in nonlinear systems [14], [32], [33]. Of special interest, the work done in [34] compares the results of EKF, UKF, and Gauss-Hermite filter 
applied to ground target tracking with particle filters. The potential benefits of UKF implementation in our system have been analyzed. This section briefly presents the basics of this filtering technique, and Section III-C will show the results of the comparison of both methods in tests performed in real scenarios.

In the UKF, it is not necessary to compute the Jacobians of the nonlinear system, although values for the sigma points must be calculated [32], [33]. Given the state vector ( $L$-dimensional) at time $k-1$, a collection of sigma points stored in the $L_{x}(2 L+1)$ matrix is computed as

$$
\begin{aligned}
\left(\mathbf{x}_{k-1}\right)_{0} & =\hat{\mathbf{x}}(k-1) \\
\left(\mathbf{x}_{k-1}\right)_{i} & =\hat{\mathbf{x}}(k-1)+(\sqrt{(L+\lambda) P(k-1)})_{i} \\
i & =1, \ldots, L \\
\left(\mathbf{x}_{k-1}\right)_{i} & =\hat{\mathbf{x}}(k-1)-(\sqrt{(L+\lambda) P(k-1)})_{i-L} \\
i & =L+1, \ldots, 2 L
\end{aligned}
$$

where

$$
\lambda=\alpha^{2}(L+\kappa)-L
$$

and $\alpha$ and $\kappa$ are scaling parameters of the filter about the scattering of the sigma points. The next step is the evolution of the sigma points through time by applying the $f$ function as

$$
\left(\mathbf{x}_{k}\right)_{i}=f\left[\left(\mathbf{x}_{k-1}\right)_{i}\right], \quad i=0, \ldots, 2 L .
$$

Finally, the computation of the state vector and its covariance matrix in the prediction step can be calculated as

$$
\mathbf{x}(k \mid k-1)=\sum_{i=0}^{2 L} W_{i}^{m}\left(\mathbf{x}_{k}\right)_{i}
$$

with

$$
\begin{aligned}
W_{o}^{m} & =\frac{\lambda}{L+\lambda} \\
W_{i}^{m} & =\frac{1}{2(L+\lambda)}, \quad i=1, \ldots, 2 L
\end{aligned}
$$

and

$P(k-1 \mid k)=\sum_{i=0}^{2 L} W_{i}^{c}\left[\left(\mathbf{x}_{k}\right)_{i}-\hat{\mathbf{x}}(k)\right]\left[\left(\mathbf{x}_{k}\right)_{i}-\hat{\mathbf{x}}(k)\right]^{T}+Q(k)$

with

$$
\begin{aligned}
& W_{o}^{c}=\frac{\lambda}{L+\lambda}+\left(1-\alpha^{2}+\beta\right) \\
& W_{i}^{c}=\frac{1}{2(L+\lambda)}, \quad i=1, \ldots, 2 L
\end{aligned}
$$

respectively, where $\beta$ is a parameter used to incorporate any prior knowledge about the distribution of $\mathbf{x}$. The tuning values used in our experiments were $\alpha=1, \kappa=0$, and $\beta=2$.
In the case of a nonlinear measurement function $h$, the transformation of the sigma points through the measurement function before the correction step must be applied by

$$
\begin{aligned}
\left(\mathbf{z}_{k}\right)_{i} & =h\left(\left(\mathbf{x}_{k}\right)_{i}\right), \quad i=0, \ldots, 2 L \\
\hat{\mathbf{z}}(k) & =\sum_{i=0}^{2 L} W_{i}^{m}\left(\mathbf{z}_{k}\right)_{i} .
\end{aligned}
$$

In our particular case, the measurement model is linear; hence, a standard Kalman estimation step will be computed.

Finally, it can be useful to augment the state vector by considering the process and measurement noises [35], although this method requires the use of additional sigma points. In our case, the process noise will be included as

$$
\mathbf{x}^{a}(k)=\left[\begin{array}{l}
\mathbf{x}(k) \\
v(k)
\end{array}\right]
$$

with its covariance defined by

$$
P^{a}(k \mid k)=\left[\begin{array}{cc}
P(k \mid k) & P_{x v}(k \mid k) \\
P_{x v}(k \mid k) & Q(k)
\end{array}\right] .
$$

\section{F. Integrity Monitoring}

As mentioned in Section I, the navigation system proposed in this paper provides two different integrity parameters. The $\mathrm{HPL}_{\mathrm{SBAS}}$ parameter can serve as an integrity parameter to evaluate the quality of a GNSS-based solution. Its value is calculated as

$$
\mathrm{HPL}_{\mathrm{SBAS}}=K_{H, \mathrm{NPA}} \cdot d_{\text {major }}
$$

where $K_{H, \mathrm{NPA}}$ is a constant that defines the kind of approach done for EGNOS (nonprecision approach in our case due to the dynamic trials performed), and $d_{\text {major }}$ is calculated by a set of operations the inputs of which are satellite geometry parameters and a variance error value of the pseudorange measurements. Further details can be found in [25]. Regardless of its utility, the HPL $\mathrm{SBAS}_{\text {B }}$ parameter subject to GPS coverage and considering only GNSS measurements cannot fully represent without interruptions the quality of the positioning provided by the whole navigation system. The provision of a parameter that completely describes the positioning quality achieved by all the sensors and without availability interruptions is highly advisable.

As previously seen, one of the main Kalman filter features is the provision of vehicle state and state covariance (matrix $P$ ). Since the first two variables of the vector state, i.e., $x$ and $y$, are, respectively, East and North coordinates of g.c., the submatrix $P_{x y}$ represents the 2-D quadratic form of the squared position error with 1-sigma scaling as

$$
P_{x y}=\left[\begin{array}{cc}
\sigma_{x}^{2} & \sigma_{x y}^{2} \\
\sigma_{x y}^{2} & \sigma_{y}^{2}
\end{array}\right] .
$$

Since $\sigma_{x}, \sigma_{y}$, and $\sigma_{x y}$ are real valued and positive, and $\sigma_{x y}<\sigma_{x}, \sigma_{y}$, thus, $P_{x y}$ is symmetric and positive definite, and 
describes an ellipse. The higher of the two eigenvalues of $P_{x y}$, i.e., $\lambda_{\max }$, which is the maximum value for the horizontal position variance, is given by

$$
\lambda_{\max }=\frac{\sigma_{x}^{2}+\sigma_{y}^{2}}{2}+\sqrt{\left(\frac{\sigma_{x}^{2}+\sigma_{y}^{2}}{2}\right)^{2}+\sigma_{x y}^{4}-\sigma_{x}^{2} \sigma_{y}^{2}} .
$$

Defining the parameter HTL as 6-sigma radius of the horizontal circle around the true position (99.99999\% of the computed fix under the assumption of scalar normal distributed errors), then its value can be calculated as

$$
\mathrm{HTL}=6 \sqrt{\lambda_{\max }}
$$

As presented, the HTL value represents the position integrity according to the filter noise estimates. Therefore, realistic vehicle models and tuning values are crucial.

\section{G. IMM-EKF Filter}

As the dynamic state of vehicles is highly variable over time, the selected model has to meet the conditions of very different situations. However, a solution based on the implementation of a unique model that fulfills the consistency requirements of scenarios with high dynamic changes provokes unrealistic noise considerations when mild maneuvers are performed, diminishing the filter efficiency and impoverishing the final solution. Therefore, an IMM filter has been developed and implemented. The IMM-EKF filter calculates the probability of success of each model at every filter execution scan, which supplies a realistic combined solution for the vehicle behavior. These probabilities are calculated according to a Markov model for the transition between maneuver states, as detailed in [30]. To implement the Markov model, it is assumed that at each scan time, there is a probability $P_{i j}$ that the vehicle will make a transition from model state $i$ to state $j$. These probabilities are assumed to be known a priori and can be expressed in a probability transition matrix such as

$$
P_{T}=\left[\begin{array}{ll}
P_{11} & P_{12} \\
P_{21} & P_{22}
\end{array}\right]=\left[\begin{array}{ll}
0.7 & 0.3 \\
0.4 & 0.6
\end{array}\right]
$$

for our case with two models $(r=2)$. Note that the sum of transition probabilities for any given state must be unity. These values are determined from the statistics of real traffic situations following [18] and are related to the mean sojourn times and sampling interval.

The likelihood calculation and the model probability update are performed according to the statistical distance value given by

$$
d^{2}=\nu^{T} S^{-1} \nu
$$

Given an IMM approach, there will be a different residual covariance matrix $S_{j}(k)$ and distance $d_{j}^{2}(k)$ associated with each of the $j$ models for the update at the scan $k$. Assuming measurement dimensionality $M$ and Gaussian statis- tics, the likelihood function for the observation model given model $j$ is

$$
\Lambda_{j}(k)=\frac{\exp \left[-d_{j}^{2}(k) / 2\right]}{\sqrt{(2 \Pi)^{M}\left|S_{j}(k)\right|}} .
$$

Using Bayes' rule, the updated model probabilities become

$$
\mu_{j}(k)=\Lambda_{j}(k) C_{j}(k-1) / C
$$

where $C_{j}(k-1)$, i.e., the probability after interaction that the vehicle is in state $j$, can be calculated as

$$
C_{j}(k-1)=\sum_{i=1}^{r} P_{i j} \mu_{i}(k-1)
$$

and the normalization constant $C$ is

$$
C=\sum_{j=1}^{r} \Lambda_{j}(k) C_{j}(k-1) .
$$

Due to the reduced number of models used, the process of data association, which is extensively discussed in the actual literature, is simplified in our case, and the state and covariance estimates can be described by [18]

$$
\begin{aligned}
\hat{\mathbf{x}}(k \mid k-1)= & \sum_{j=1}^{r} C_{j}(k-1) \hat{\mathbf{x}}_{j}(k \mid k-1) \\
P(k \mid k-1)= & \sum_{j=1}^{r} C_{j}(k-1) \\
& \times\left[P_{j}(k \mid k-1)+\left(\hat{\mathbf{x}}(k \mid k-1)-\hat{\mathbf{x}}_{j}(k \mid k-1)\right)\right. \\
& \left.\cdot\left(\hat{\mathbf{x}}(k \mid k-1)-\hat{\mathbf{x}}_{j}(k \mid k-1)\right)^{T}\right] .
\end{aligned}
$$

\section{H. Hardware Architecture}

The hardware architecture of the OBE is based on a standard single-board computer with a 32-bit Pentium processor. The vehicle PC interacts with the user via the human-machine interface by a monitor, keyboard, and mouse. Serial buses communicate the sensors with the PC via RS232 and the Controller Area Network bus. Some other additional communication networks are also available. A BlueTooth wireless link can be used to connect the vehicle PC with a laptop or other mobile devices such as personal digital assistants, PocketPCs, etc. A wireless local area network connection is available through the Personal Computer Memory Card International Association slot of the vehicle central processing unit, which facilitates communication with nearby vehicles. Finally, a General Packet Radio Service/Universal Mobile Telecommunications System (GPRS/UMTS) link supplies Internet connection to the system. The GPRS/UMTS link is used for receiving the EGNOS messages via SISNeT [11] and can also be used to communicate the vehicle with remote stations (or other vehicles) for locationbased services. The test vehicle prototype is shown in Fig. 3. 


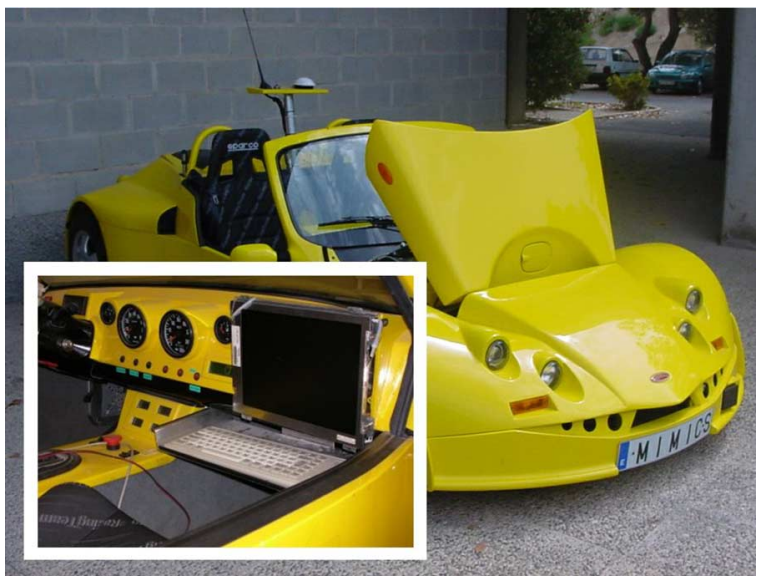

Fig. 3. Test vehicle prototype.

\section{Conclusions of the System Description}

Compared to traditional approaches of the current literature in Section III, the main contributions of this paper are summarized as follows:

1) the capability of the proposed system to solve the typical problems of traditional low-cost navigation systems based on dead reckoning (DR), such as those derived from spurious observations and GPS outages;

2) the potential benefits of UKF as compared with the EKF in our case of study;

3) the IMM-EKF performance compared with single-model (SM) implementations, especially in changing maneuvering environments;

4) the possible improvements of using double-constellation GPS/GLObalnaya NAvigatsionnaya Sputnikovaya Sistema (GLONASS) receivers and the European SBAS (EGNOS/SISNeT) for both localization and integrity information provision;

5) the suitability of the HTL parameter to represent the integrity of a GPS/INS integrated system, avoiding the disadvantages of exclusively GNSS-based solutions, along with the appropriate noise estimation supplied by the IMM-EKF

\section{TRIALS AND Results}

Concerning road applications of the GNSS sensors, two different scenarios must be distinguished. Despite the fact that objectives and technologies are the same, the different problems a GNSS sensor has to deal with in urban and wide open environments encourage their study from different points of view [36]. Whereas satellite constellation visibility in wide open environments is not a problem, and our main efforts are focused on diminishing the pseudorange errors and increasing the positioning accuracy, in urban environments, the signal availability and the multipath propagation that provokes spurious GPS positions due to the signal reflection near the antenna are the main problems.

To evaluate the performance of our system in dynamic tests, depending on the circuit features, different references can be used. In the trials performed in closed circuit, a custom-made
TABLE I

Main Features of the Test Scenarios

\begin{tabular}{|c|c|c|c|}
\hline Sc. & Circuit & Data & Filter \\
\hline 1 & Highway & GPS/GLONASS & No Filter \\
\hline 2 & City & GPS/GLONASS & No Filter \\
\hline 3 & Static & GPS/EGNOS & No Filter \\
\hline 4 & Combined & GPS/EGNOS/SISNeT & No Filter \\
\hline 5 & Campus & GPS/INS GPS outages & DR/IMM-EKF (NV) \\
\hline 6 & Campus & GPS/INS GPS outages & IMM-EKF \\
\hline 7 & Campus & GPS/INS Long GPS gap & IMM-EKF \\
\hline 8 & Combined & GPS/INS/Odo & EKF \\
\hline 9 & Combined & GPS/INS/Odo & UKF \\
\hline 10 & Campus & Odo/INS - No GPS & SM-EKF/IMM-EKF \\
\hline 11 & Campus & GPS/INS GPS outages & IMM-EKF \\
\hline
\end{tabular}

TABLE II

Thales GG24 Performance Parameters (Thales Specifications)

\begin{tabular}{|l|l|}
\hline R.T. Position (Autonomous) & CEP: $3.2 \mathrm{~m}(10.50 \mathrm{ft}$.) \\
\hline R.T. Position (Differential) & CEP: $40.0 \mathrm{~cm}(15.75 \mathrm{in})$. \\
\hline Velocity & $0.1(95 \%)(\mathrm{knots})$ \\
\hline
\end{tabular}

map developed by using the Trimble Pathfinder Office version 3.0. package provides $30-\mathrm{cm}$ accuracy of the map reference [differential global positioning system (DGPS)]. In those cases where DGPS measurements were not available, EGNOS has been selected as the best possible reference. For Geostationary Earth Orbit (GEO)/GPS dark areas where EGNOS is not available (not even via SISNeT), Navteq maps can be used for visual inspection of the positioning.

Trials are organized according the features of interest in different scenarios, summarized in Table I, and explained in following sections.

\section{A. GPS/SBAS Trials}

1) Double Constellation Trials: For the tests performed in scenarios 1 and 2, the GPS/GLONASS Thales GG24 sensor was used. The performance parameters of this sensor are shown in Table II.

Fig. 4 shows a comparison of the cumulative distribution functions (cdfs) of the HDOP value in different tests performed in a wide open environment (scenario 1) and an urban area (scenario 2). Assuming that $\mathrm{HDOP}=3$, the signal availability in the urban area is not much higher than $80 \%$ (in the best case). However, values of $95 \%$ are usual in the highway scenario. These values are similar to those obtained by using a single GPS sensor. According to our tests, despite the fact that there is a slight increase of the position availability by using the double constellation sensor, the main problems, such as lack of coverage in city environments and multipath errors remain.

2) SBAS Improvements: In order to determine the accuracy improvements obtained by using EGNOS/SISNeT, the sensor selected was Novatel OEM-3. Its performance parameters are shown in Table III.

In this case, the GPS/EGNOS antenna was placed in a wide open area with very good geostationary and GPS signal visibility (scenario 3). As can be seen in Fig. 5, when using a low-cost GPS receiver, the HPE95 value increases to $8.1 \mathrm{~m}$., while the Circle Error Probable (CEP) value $=3.2 \mathrm{~m}$ for 3694 fixes (a CEP of $3.2 \mathrm{~m}$ is interpreted to mean that $50 \%$ of the position estimates will be within $3.2 \mathrm{~m}$ of the actual position). Despite the fact that most of the HPE95 values surround the 


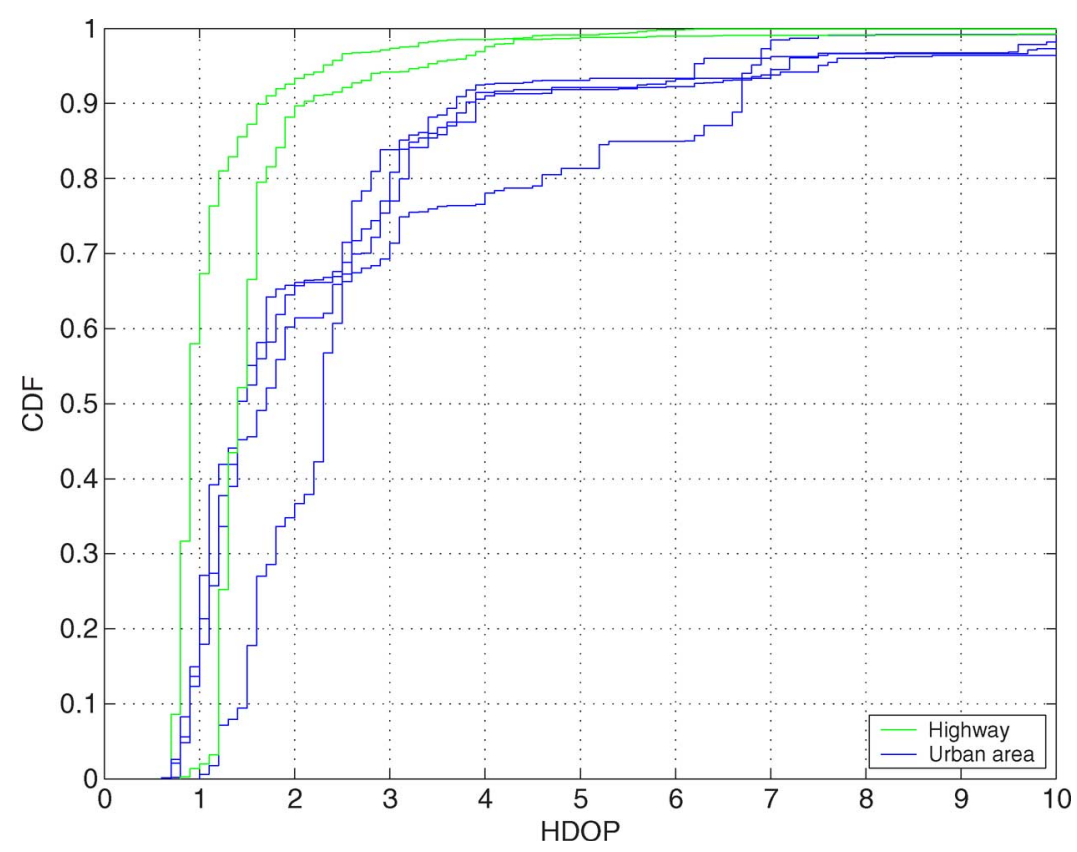

Fig. 4. Comparison of the cdf of the HDOP values logged by the Thales GG24 sensor in (scenario 1: solid green), a wide open area, and (scenario 2: solid blue) an urban environment.

TABLE III

Novatel OEM-3 Performance PARAMETERS (NOVATEL SPECIFICATIONS)

\begin{tabular}{|l|l|}
\hline Single Point (L1) & CEP: $1.8 \mathrm{~m} .(5.9 \mathrm{ft}$ ) \\
\hline Single Point (L1/L2) & CEP: $1.5 \mathrm{~m} .(4.92 \mathrm{ft})$. \\
\hline SBAS L1 & CEP: $1.2 \mathrm{~m} .(3.94 \mathrm{ft})$ \\
\hline SBAS L1/L2 & CEP: $0.8 \mathrm{~m} .(2.62 \mathrm{ft})$. \\
\hline
\end{tabular}

$3 \mathrm{~m}$, many of them appear close to $8 \mathrm{~m}$. In the same test, applying EGNOS corrections to the GPS positions, the HPE95 value decreases to $5.2 \mathrm{~m}$, and CEP $=1.9 \mathrm{~m}$ (Fig. 6). Similar results were achieved in several tests. Therefore, EGNOS can increase the accuracy of the GPS positions.

Table IV summarizes the results obtained in dynamic tests regarding the availability of the EGNOS/SISNeT corrections in test scenario 4. According to these results, EGNOS can increase the accuracy of single GPS positions during $64.78 \%$ of the traveled time in the combined circuit tested. By using SISNeT and EGNOS corrections via the Internet, this value can be increased from $64.78 \%$ up to $89.15 \%$ (which is $24.37 \%$ more). However, despite of the important improvements, high demanding applications require more than $96 \%$ of positioning availability, and some assistance positioning systems are recommended, especially in city environments, where this rate decreases to $60 \%$.

\section{B. GPS/SBAS/INS Performance}

The lack of GPS coverage in some environments is a considerable problem that cannot be solved by a GPS. As observed in the previous section, the use of SISNeT and EGNOS in Europe improves the single GPS solution quality but cannot guarantee system success during the outages of the GPS signal. The performance of the GPS/SBAS/INS integrated system developed is commented next. Main issues analyzed in this section are fault detection capacity, continuous positioning availability, and the ability to reproduce the vehicle behavior in different usual driving situations.

The INS was based on an MT9-B Xsens IMU, the performance parameters of which are shown in Table $\mathrm{V}$.

1) Fault Detection: In order to achieve a reliable solution, system failures must be detected, and proper actions must be performed. The term fault includes not only hardware and software failures but also false measurements coming from the sensors. First, acceptation must be considered in the system designing phase. In this section, we will focus our attention on the problem of false measurements.

Spurious positions are one of the main problems for a highintegrity navigation system, especially in city environments. Spurious (or false) measurements can be defined as those outside of the $6 \sigma$ scaling certainty region centered in the real value of the measurement (assuming Gaussian distributions). Unfortunately, real values are unknown, and some other parameters must be used to categorized a measurement as false.

For spurious detection in GNSS sensors, as previously commented, the RAIM technique requires six tracked satellites to detect and reject an anomaly. The use of SBAS can improve this situation but only when geostationary satellite is in view. Nevertheless, the use of information redundancy from all the sensors can effectively help remove spurious measurements from the solution and not exclusively from satellite anomalies. In our filter, a validation algorithm based on the Nyquist inequation (8) has been included to achieve this.

Fig. 7 shows the trajectories logged by our navigation system during the tests performed in scenario 5, i.e., the Campus of Espinardo, University of Murcia. The black line represents the custom-made DGPS reference, the blue dots represent the GPS/EGNOS positions, the green line represents the DR solution, and the red line represents the filter outcome (noted 


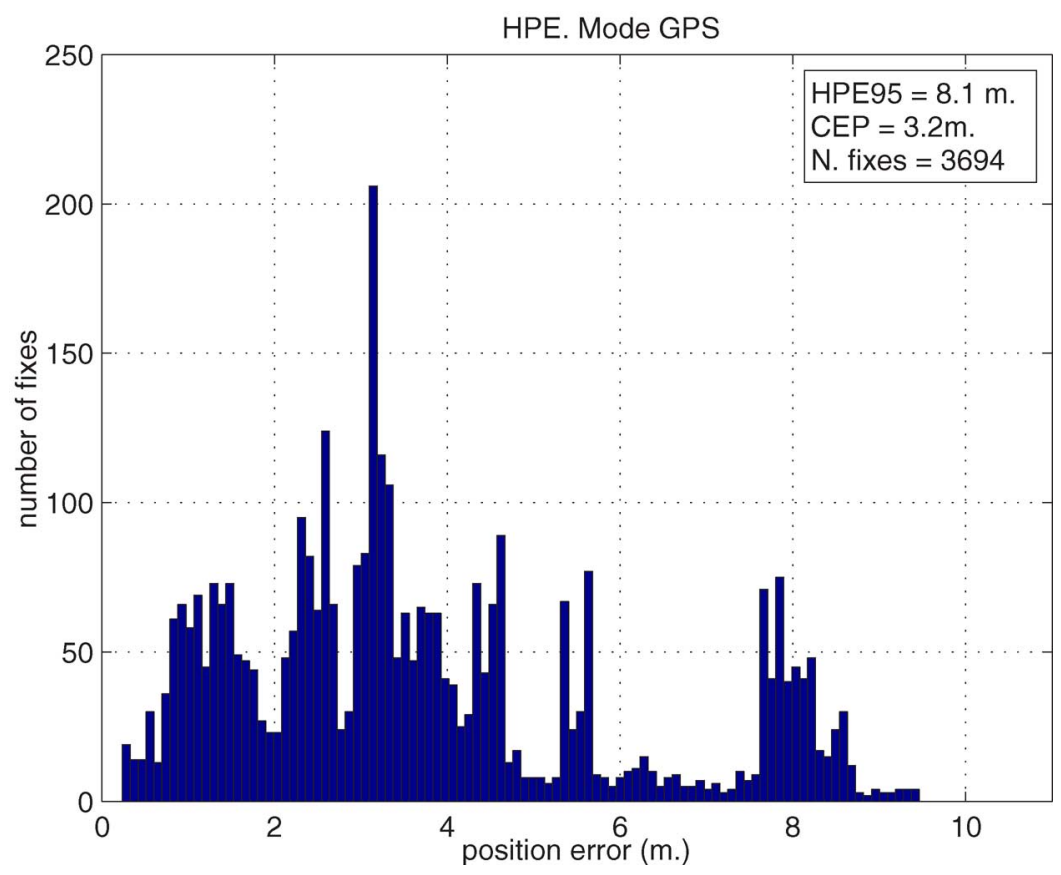

Fig. 5. HPE, CEP, and number of fixes by using GPS in test scenario 3 .

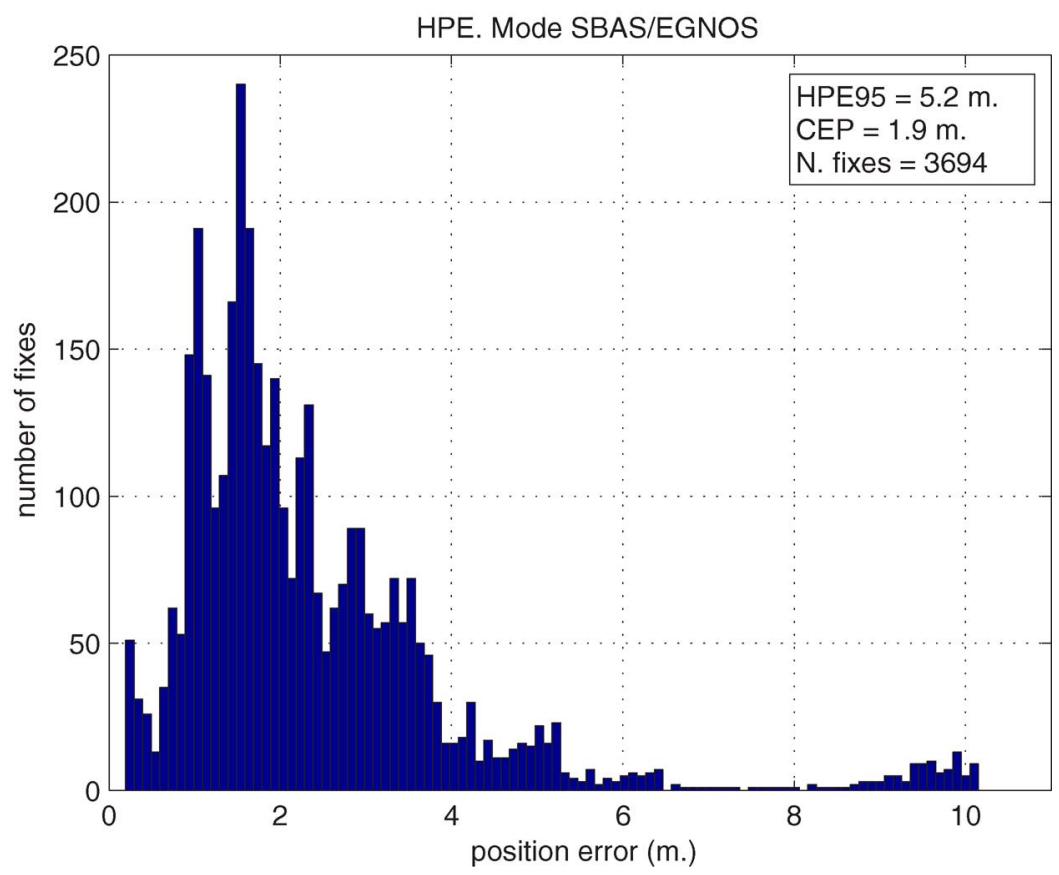

Fig. 6. HPE, CEP, and number of fixes by using EGNOS in test scenario 3.

TABLE IV

EGNOS/SISNeT AVAILABILITY IN TEST SCENARIO 4

\begin{tabular}{|c|c|c|c|c|c|}
\hline N. Fixes & No Pos. & GPS & EGNOS & SISNeT & Distance \\
\hline 2777 & $3.78 \%$ & $7.05 \%$ & $64.78 \%$ & $24.37 \%$ & $20,07 \mathrm{Km}$. \\
\hline
\end{tabular}

by IMM-EKF in the legend). During this semiurban path, no position would be available during the frequent GPS outages. Besides, a single GNSS solution would offer false position information where spurious GPS positions are logged.

Fig. 8 shows a GPS outage period with detail. Traditional low-cost solutions for the outages of GPS signal are based on DR. In DR, no data fusion is performed, and the position
TABLE V

MT9-B Xsens Performance Parameters (Xsens Specifications)

\begin{tabular}{|l|l|l|}
\hline Parameter & $\begin{array}{l}\text { Rate of turn } \\
(\mathrm{deg} / \mathrm{s} .)\end{array}$ & $\begin{array}{l}\text { Acceleration } \\
\left(\mathrm{m} / \mathrm{s}^{2} .\right)\end{array}$ \\
\hline Full Scale (units) & $+/-900$ & $+/-20$ \\
\hline Linearity (\% of FS) & 0.1 & 0.2 \\
\hline Bias stability (units 1- $\sigma)$ & 5 & 0.02 \\
\hline Scale factor stability $(\% 1-\sigma)$ & - & 0.05 \\
\hline Noise (units RMS) & 0.7 & 0.01 \\
\hline Alignment error (deg) & 0.1 & 0.1 \\
\hline
\end{tabular}

is supplied by odometry measurements and the orientation value coming from the electronic compass during the GPS gaps. Due to sensor errors and model constraints, the position 


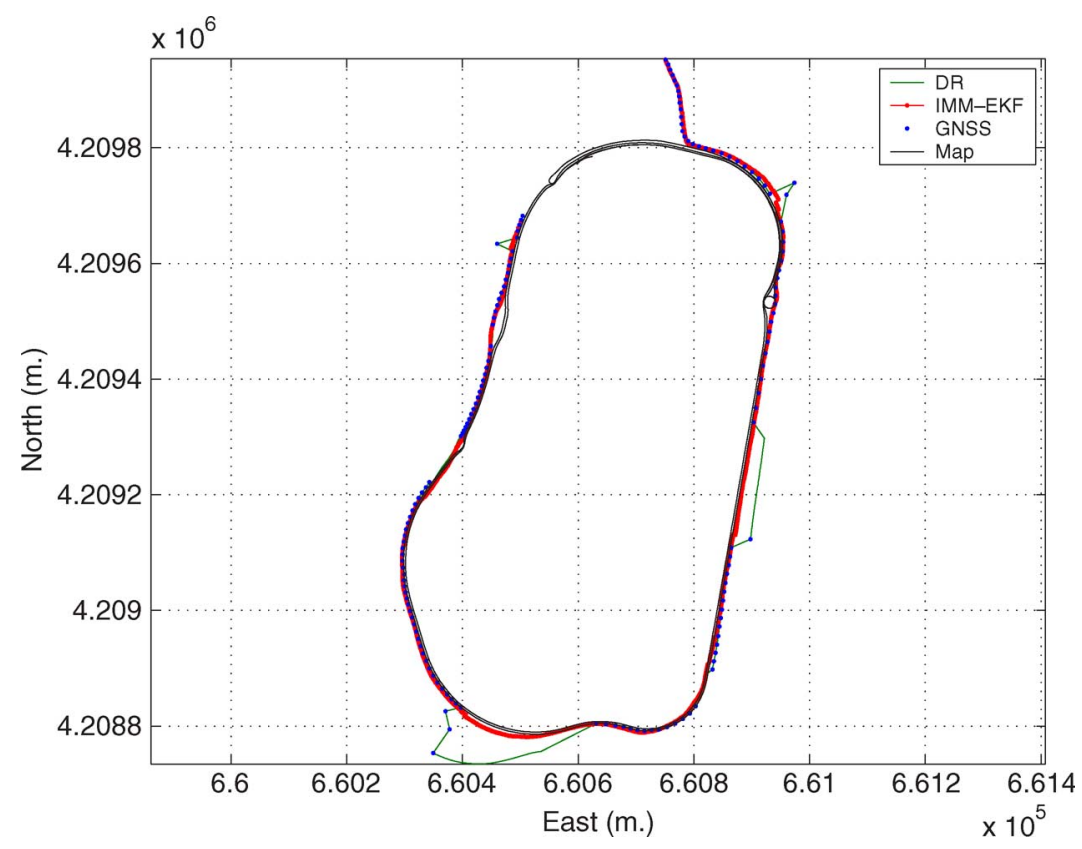

Fig. 7. Estimated vehicle trajectories in test scenario 5 without applying validation phase.

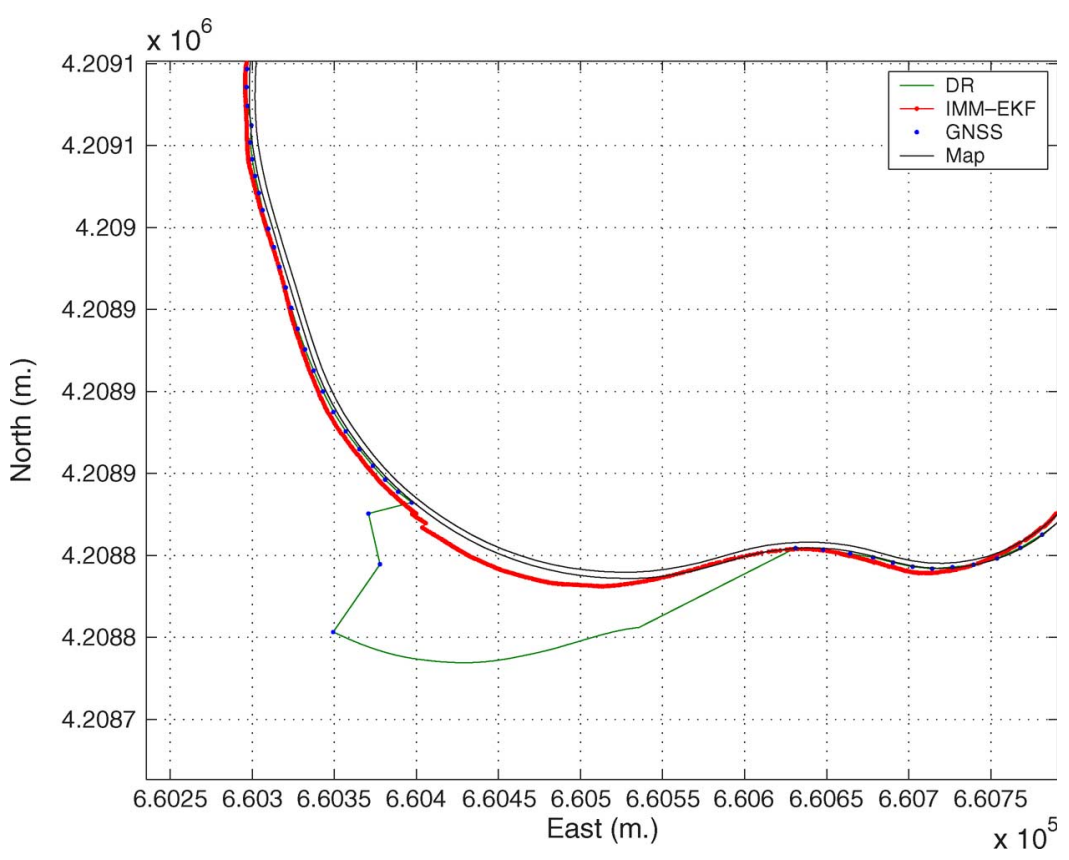

Fig. 8. Detail of the estimated vehicle trajectory in test scenario 5 without applying validation phase.

error increases, and undesirable drifts as a consequence of slides, bad velocity, and heading estimation, and inaccurate wheel diameter are not corrected. In the lower part of Fig. 8, a typical situation with GPS coverage gap just after spurious measurements is visible. This situation, which is very usual in built-up environments where the signal quality decreases before disappearing completely, provokes undesirable results by using the DR solution. As visible, the IMM-EKF solution softens the multipath propagation errors, but undesirable trimmings still appear due to multipath spurious measurements. In this first approach, no validation algorithm was implemented.

Fig. 9 presents the results obtained in test scenario 6 . During the test, the conditions were similar to those in test scenario 5 , but in this trial, the validation algorithm described in Section II-D2 was applied. As visible, the implemented algorithm rejects the spurious GPS positions from the solutions, avoiding unpleasant drifts in the positioning consequence of spurious GPS measurements.

2) Continuous Accurate Positioning: What most users desire, even more than high accuracy in the positioning system, is continuous accurate positioning. The developed IMM-EKF system must provide continuous accurate position also during gaps of GPS coverage.

Figs. 7-10 illustrate typical situations in urban environments, where problems with GPS/GEO coverage and multipath effects are usual. The capability of the system to provide continuous 


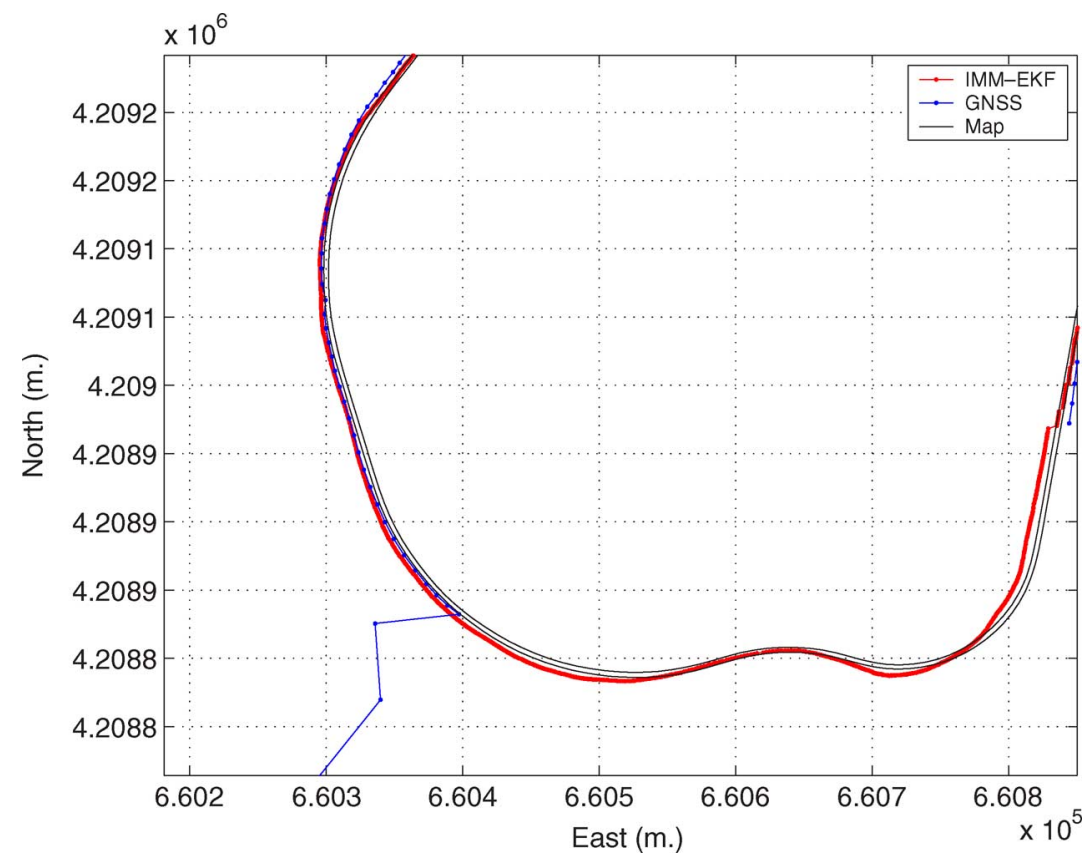

Fig. 9. Detail of the estimated vehicle trajectory in test scenario 6 applying validation phase.

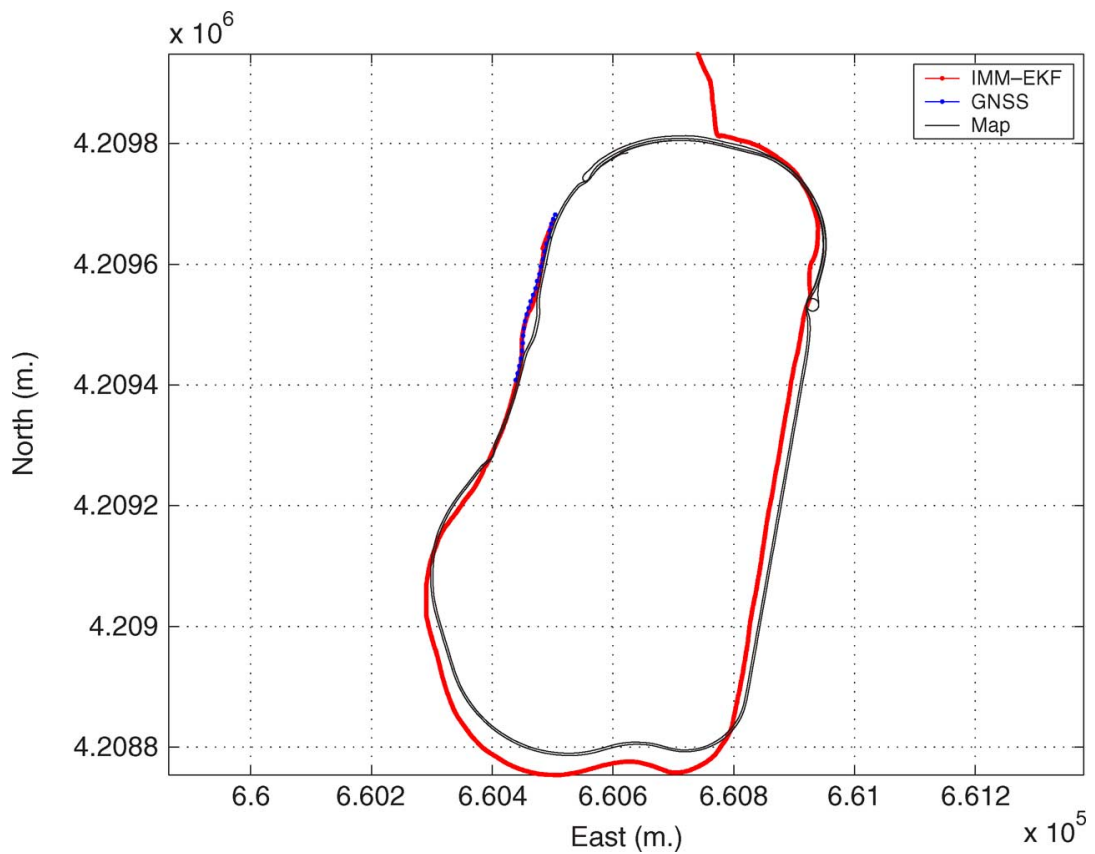

Fig. 10. Estimated vehicle trajectories in test scenario 7.

positioning is visible. Figs. 11-14 show the position estimate errors during trials performed in scenarios 5-7. In Fig. 11, the difference between the DR position estimate error and the IMM-EKF achieved is clearly visible. As can be seen in Fig. 12, although the position error estimate of the DR approach is smaller during periods with GPS/GEO visibility, the value of the Euclidean distance (ED) when the GPS signal is lost can increase up to $50 \mathrm{~m}$ (as compared to estimated path truth). In the same test, and using the IMM-EKF solution, the value of the ED remained below $10 \mathrm{~m}$ in the worst case. In test scenario 6, values of the ED lower than $5 \mathrm{~m}$ are usual with peaks of $15 \mathrm{~m}$ after $42 \mathrm{~s}$ of GPS unavailability. Fig. 14 presents the position estimate errors for scenario 7 (Fig. 10). In this test, which was done to evaluate the behavior of the system during long GPS gaps, the values of the ED reached $35 \mathrm{~m}$ after $5 \mathrm{~min}$ and $2.5 \mathrm{~km}$ with null satellite visibility, which is good enough to fulfill our requirements. Table VI shows a comparison of the root mean square (rms) value in the different scenarios presented, as referred to the GPS unavailability. RMS values for position estimate errors below $5 \mathrm{~m}$ can be considered as suitable for most of the actual navigation applications. On the other hand, a value of $17.5 \mathrm{~m}$ after 5 min and $2.5 \mathrm{~km}$ of GPS unavailability can be considered good. 

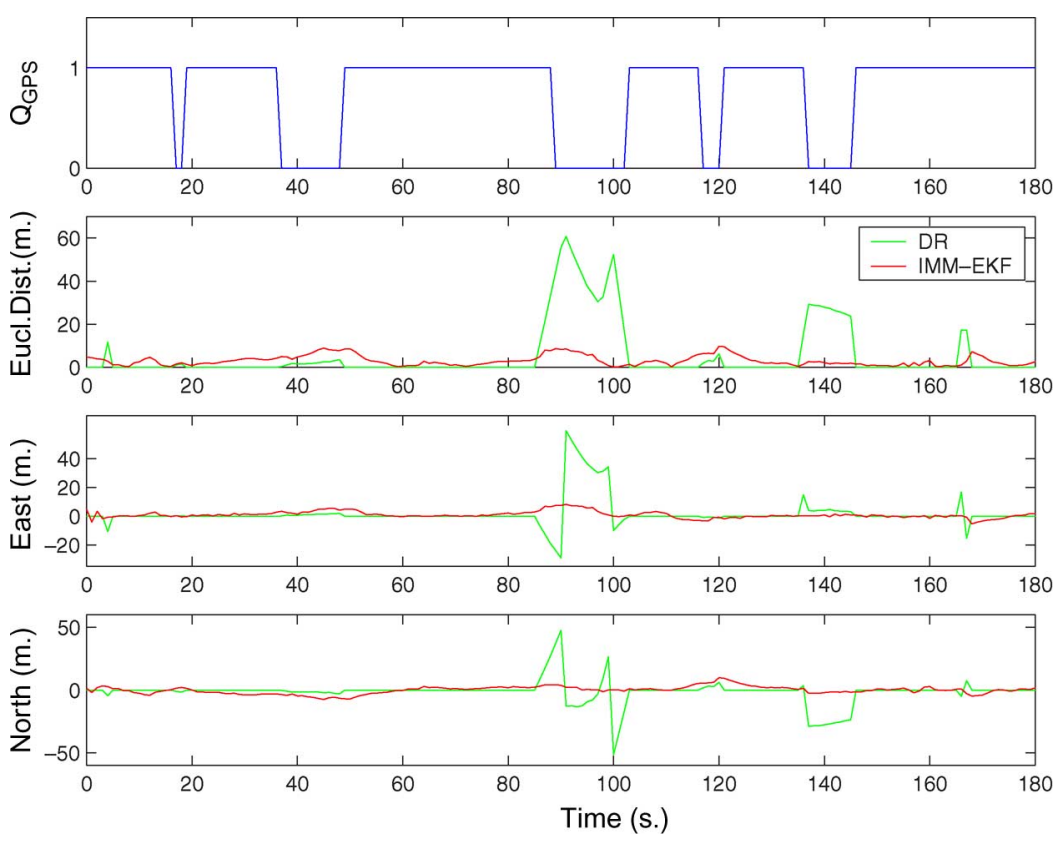

Fig. 11. Comparison of the position error estimate obtained by DR method and the IMM-EKF in the test performed in scenario 5.
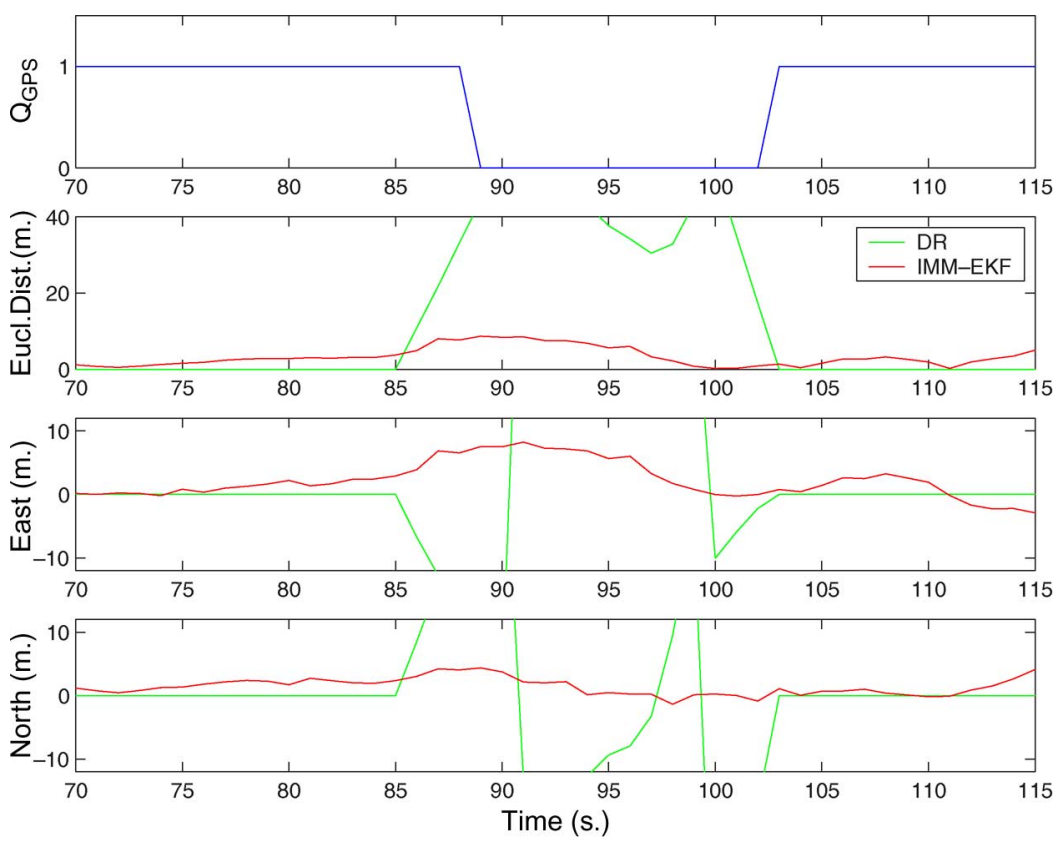

Fig. 12. Detail of the comparison of the position error estimate obtained by DR method and the IMM-EKF in the test performed in scenario 5.

In the tests performed in scenarios $5-7$, no sharp turns and abrupt maneuvers were performed; hence, the study of the IMM method has no special interest. This issue will be explored in Section III-D.

\section{Comparison of UKF and EKF Performances}

In this section, a comparison of the EKF (scenario 8) and UKF (scenario 9) performances will be done. To accomplish this, tests were realized in a controlled combined circuit with precise custom-made map information available. Both filters were run with the same data sets, obtaining each time similar results as those presented in this paper. During the $3-\mathrm{km}$ test, an average velocity of $40 \mathrm{~km} / \mathrm{h}$ was recorded, and GPS outages of $50 \mathrm{~s}$ were simulated.

Table VII summarizes the estimated maximum and rms errors for the tests performed in this circuit with both EKF and UKF. The errors and noise estimations obtained by both methods during the test can be seen in Figs. 15 and 16 (in detail). The achieved results do not present any significant improvement by using the UKF implementation in the test performed, and no special benefit is obtained of UKF.

1) Conclusions of the UKF-EKF Implementation: The UKF approach has been presented in the recent literature as an improvement of the deficiencies of the EKF due to the nonlinearities of the system. However, both KF and UKF rely 

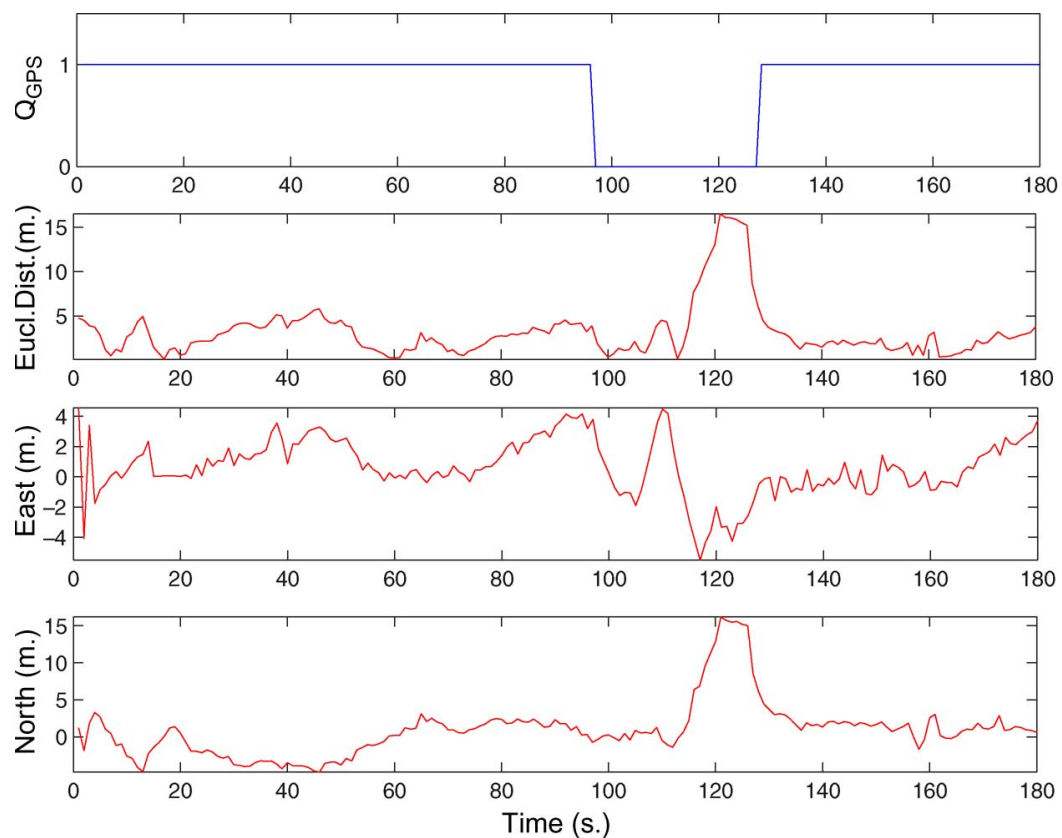

Fig. 13. Position error estimate obtained by IMM-EKF in the test performed in scenario 6.
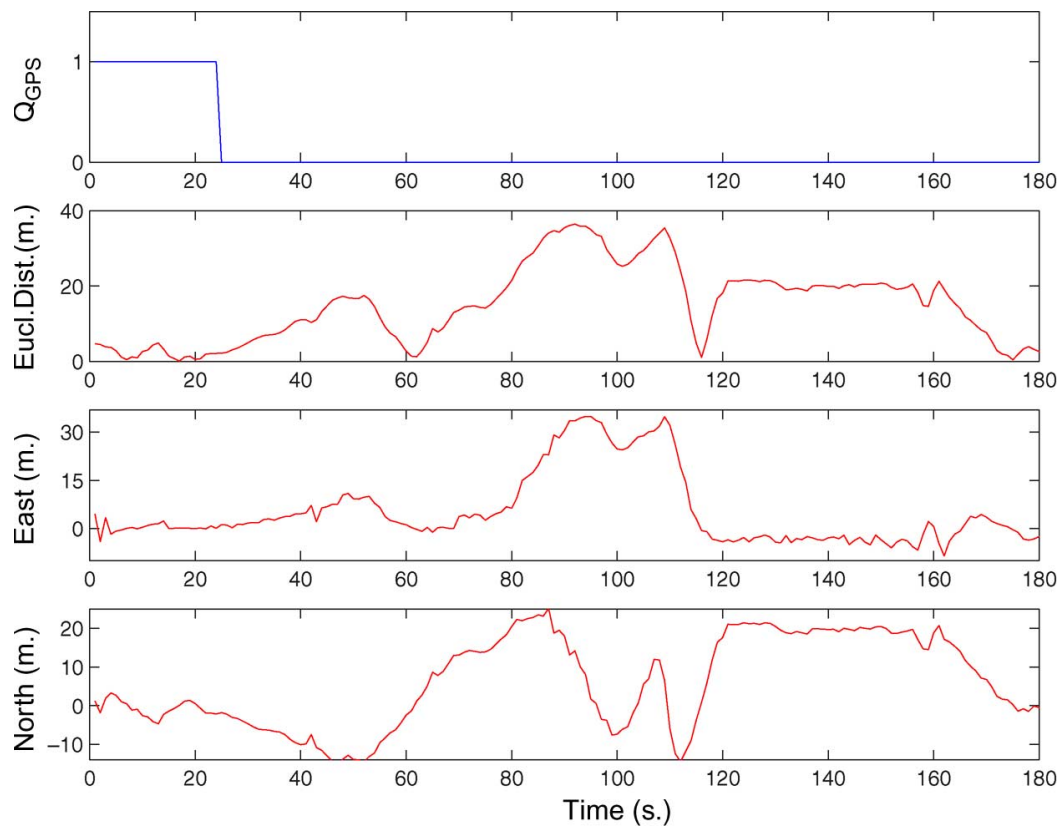

Fig. 14. Position error estimate obtained by IMM-EKF in the test performed in scenario 7.

TABLE VI

GPS OUTAGES AND RMS VALUES FOR POSITION ESTIMATE ERRORS IN SCENARIOS 5-7

\begin{tabular}{|c|c|c|c|c|}
\hline Scenario & 5: DR & 5: IMM & 6: IMM & 7: IMM \\
\hline RMS & $13.6(\mathrm{~m})$. & $3.7(\mathrm{~m})$. & $4.2(\mathrm{~m})$. & $17.5(\mathrm{~m})$. \\
\hline GPS outage & $35(\mathrm{~s})$. & $35(\mathrm{~s})$. & $42(\mathrm{~s})$. & $5(\mathrm{~min})$. \\
\hline
\end{tabular}

on an appropriate definition of the vehicle model to properly perform, and the use of simple models does not provide good results in challenging scenarios, while high dynamic models lead to the impoverishment or the complete divergence of the solution when wrongly applied. The results obtained in
TABLE VII

GPS OUtages, MAXimum, AND RMS VALUes For Position ESTIMATE ERRORS IN SCENARIOS 8 AND 9

\begin{tabular}{|c|c|c|}
\hline Scenario & 9: UKF & 5: EKF \\
\hline RMS & $4.52(\mathrm{~m})$. & $4.51(\mathrm{~m})$. \\
\hline Maximum & $16.25(\mathrm{~m})$. & $16.16(\mathrm{~m})$. \\
\hline GPS outage & $50(\mathrm{~s})$. & $50(\mathrm{~s})$. \\
\hline
\end{tabular}

the test performed present no significant differences between both implementations. Due to that, the most widely used EKF has been selected for the IMM method. Nevertheless, due to its potential benefits in diminishing nonlinearity errors, future 


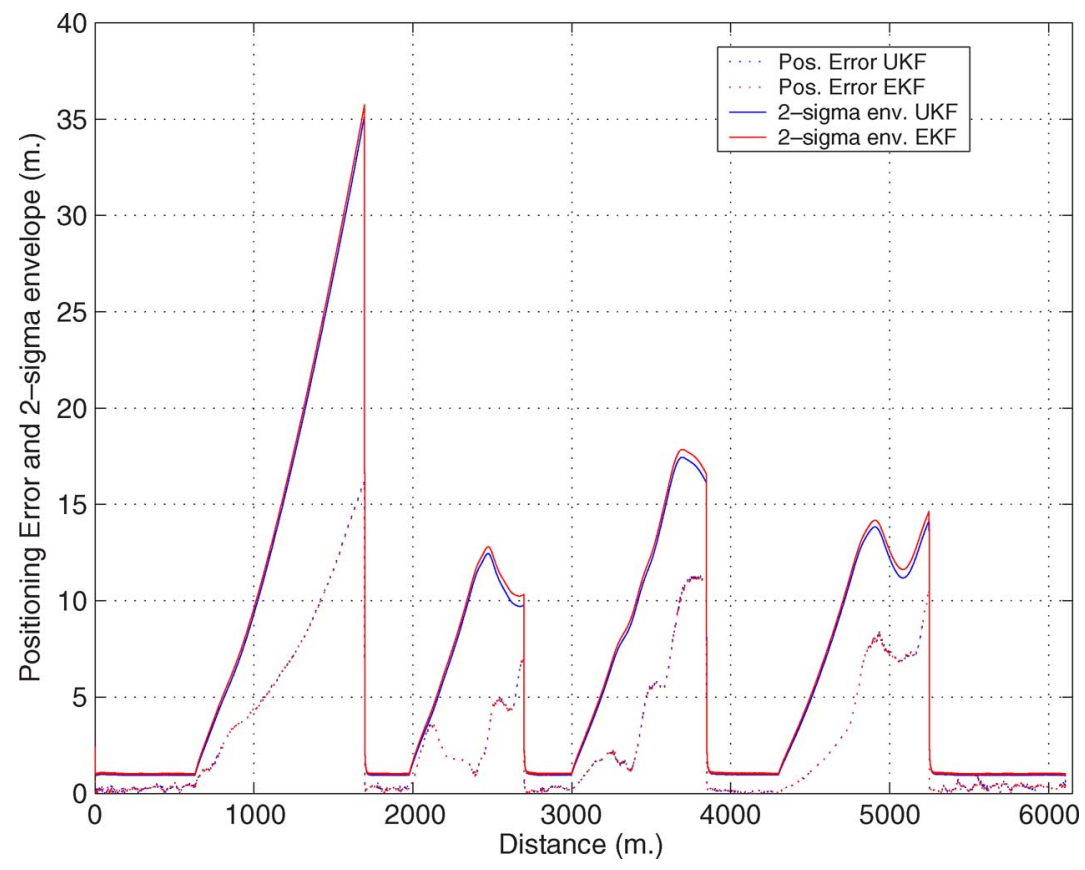

Fig. 15. Comparison of position error estimate and 2-sigma envelope obtained by (scenario 8) EKF and (scenario 9) UKF.

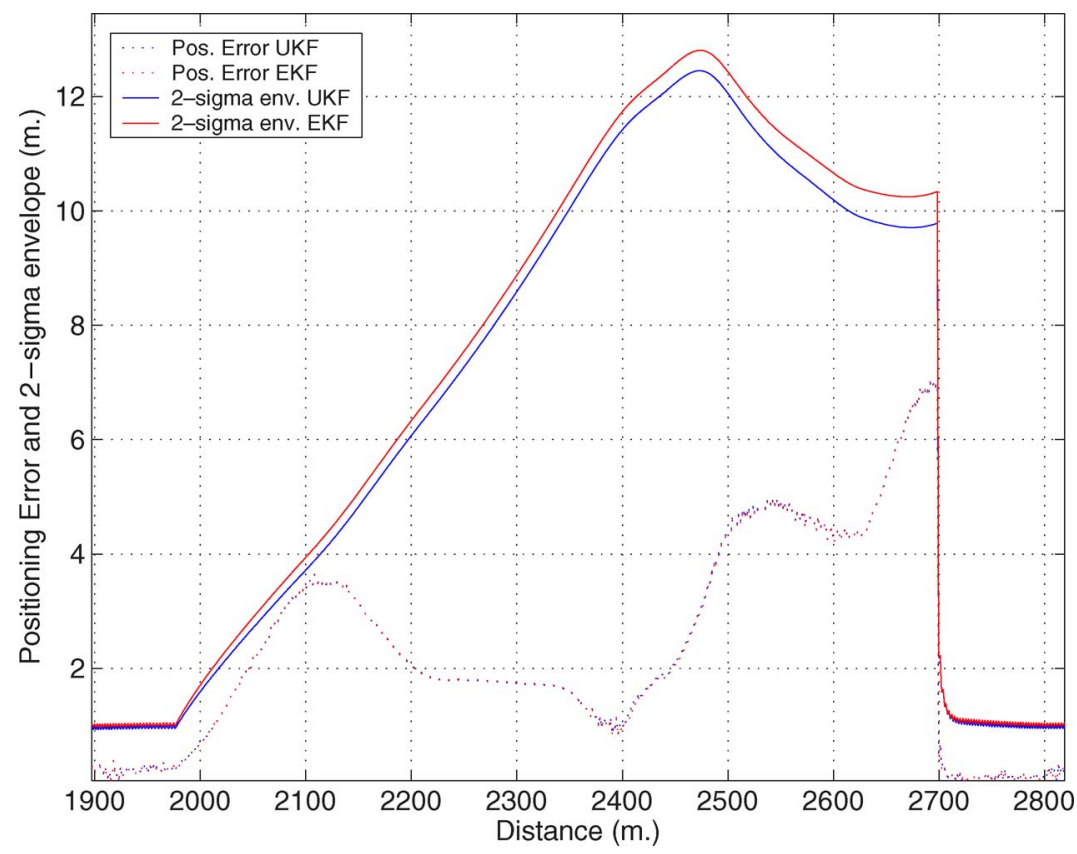

Fig. 16. Detail of the comparison of position error estimate and 2-sigma envelope obtained by (scenario 8) EKF and (scenario 9) UKF.

investigations in vehicle models and filtering techniques will also consider the UKF option.

\section{Trials With Abrupt Maneuvers}

In scenarios with low dynamic changes such as highways, a model representing a straight trajectory as presented in this paper works correctly. However, when sharp turns and abrupt maneuvers are performed, the SM approach cannot properly represent the behavior of the vehicle, and the use of a curved trajectory model is advisable. Unfortunately, the assumption of every movement as a curve provokes an increase in the noise considerations, having repercussions on the position quality. The IMM-EKF filter implemented runs both filters (straight and curved) in parallel, estimating the probabilities of defining the vehicle behavior for both models and offering a unique common solution by mixing both filtering processes according to the movement features at every scan.

In the test performed in scenarios 5-7, and 11, no sharp turns were done. Hence, no special benefit has been obtained in using the IMM-based method. The probability of the straight model during the whole trajectory remains close to unity. Therefore, no further IMM considerations will be done with regard to these scenarios. Since sharp turns and abrupt maneuvers are 


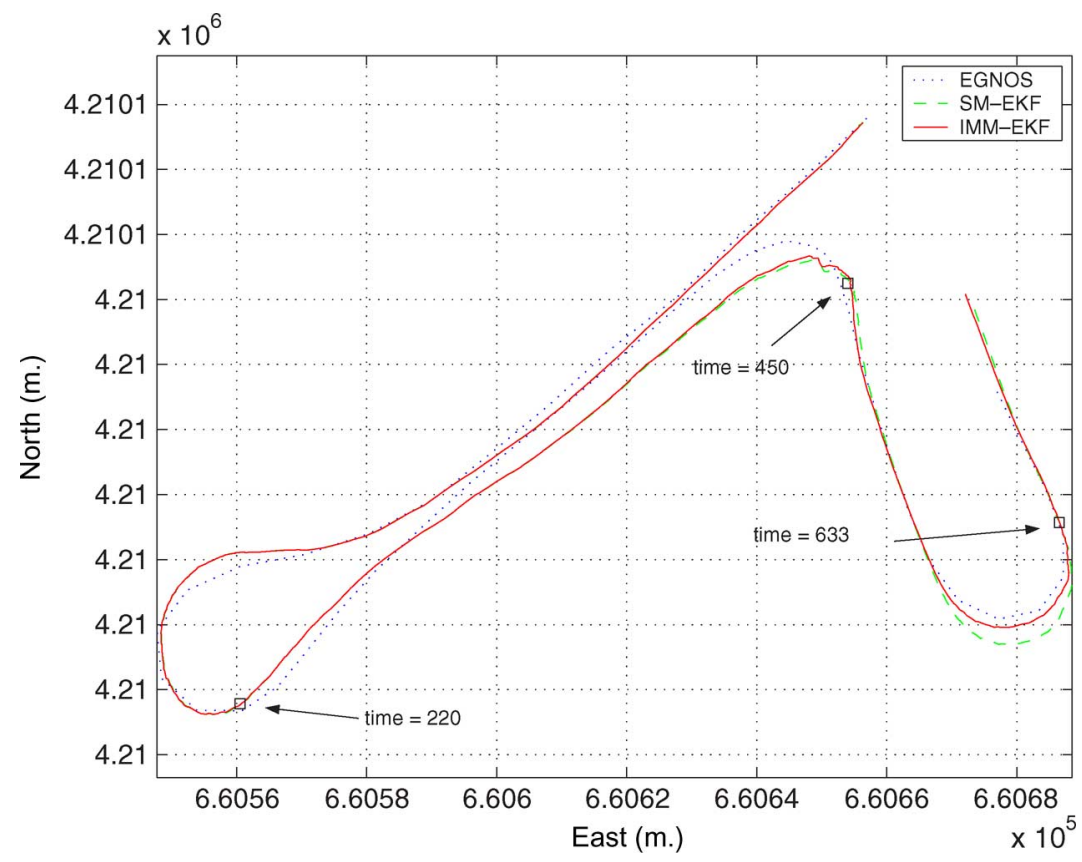

Fig. 17. Comparison of SM-EKF and IMM-EKF trajectories obtained in the test performed in scenario 10.

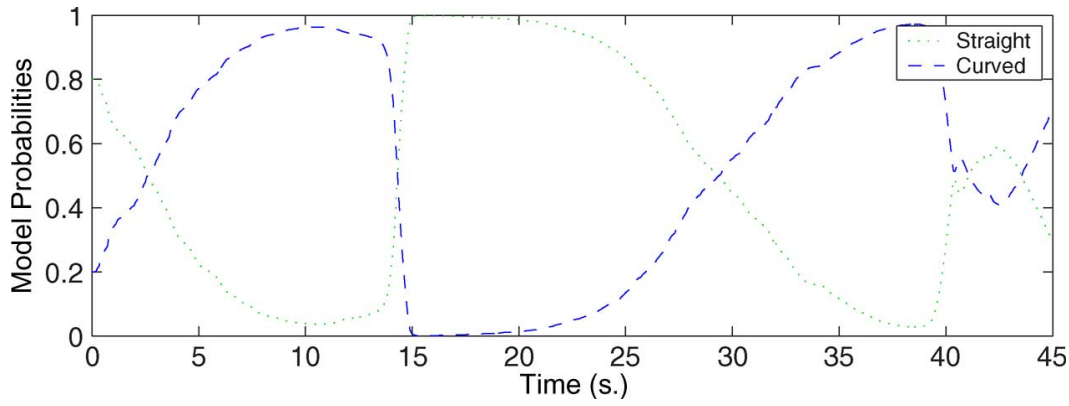

Fig. 18. Model probabilities in test performed in scenario 10.

usually performed in short-distance situations such as urban environments or indoor maneuvering, it is assumed that no GNSS information is supplied to the filter during the trials performed in test scenario 10 .

In Fig. 17, a comparison between the trajectory offered by the IMM-EKF filter and the SM solution in this scenario is shown. The nature of the Markov transition process generates the switching aspect of the IMM-EKF solution, where the periods of dominance of one model correspond to high probability values for this model. Fig. 18 presents the values of the model probabilities during the trajectory. The relation between the model probability values and the IMM solution can be appreciated. According to this figure, key points for the probability values correspond to time values of 220,450 , and $633 \mathrm{~s}$. The vehicle positions at those instants are marked in the trajectory shown in Fig. 17. As observed, those values correspond to changes in the maneuver state, which changes from a curved trajectory to a straight one and vice versa.

Since real trials were performed, no certain values for vehicle positions were available. The nature of the trials for the tests performed in scenario 10 differs from the proper maneuvering required to develop accurate DGPS references. In this situation, we consider EGNOS as the best estimator of the truth path.
The results of the position estimate errors for the trajectory performed in test scenario 10 (Fig. 17) by using an SM filter (SM-EKF) and the implemented IMM-EKF can be seen in Fig. 19. A CEP value of $3 \mathrm{~m}$ for the EGNOS reference must be considered. As can be seen, when using the IMM-EKF approach, the value of the ED is smaller than in the SM-EKF case, which remains below $3 \mathrm{~m}$ during the whole trajectory. Table VIII shows a comparison of the rms values for both SM and IMM solutions. In a test of only $390 \mathrm{~m}$, a reduction of $0.484 \mathrm{~m}$ is achieved by using the IMM-EKF implementation.

\section{E. System Integrity Monitoring}

Fig. 20 shows the trajectory of test scenario 11 , which was selected to test the suitability of the HTL parameter. In this typical semiurban scenario, no integrity information would be available during the frequent periods of GPS outage. Additionally, the geostationary satellite is often missed, still with enough GPS satellites in view. Therefore, SISNeT would be required to guarantee the provision of HPL SBAS $_{\text {wh }}$ wPS is available.

In this test performed in controlled scenario 11, GPS and EGNOS outages were simulated. This allows testing the position results and the provision of the ED of the filter solution 

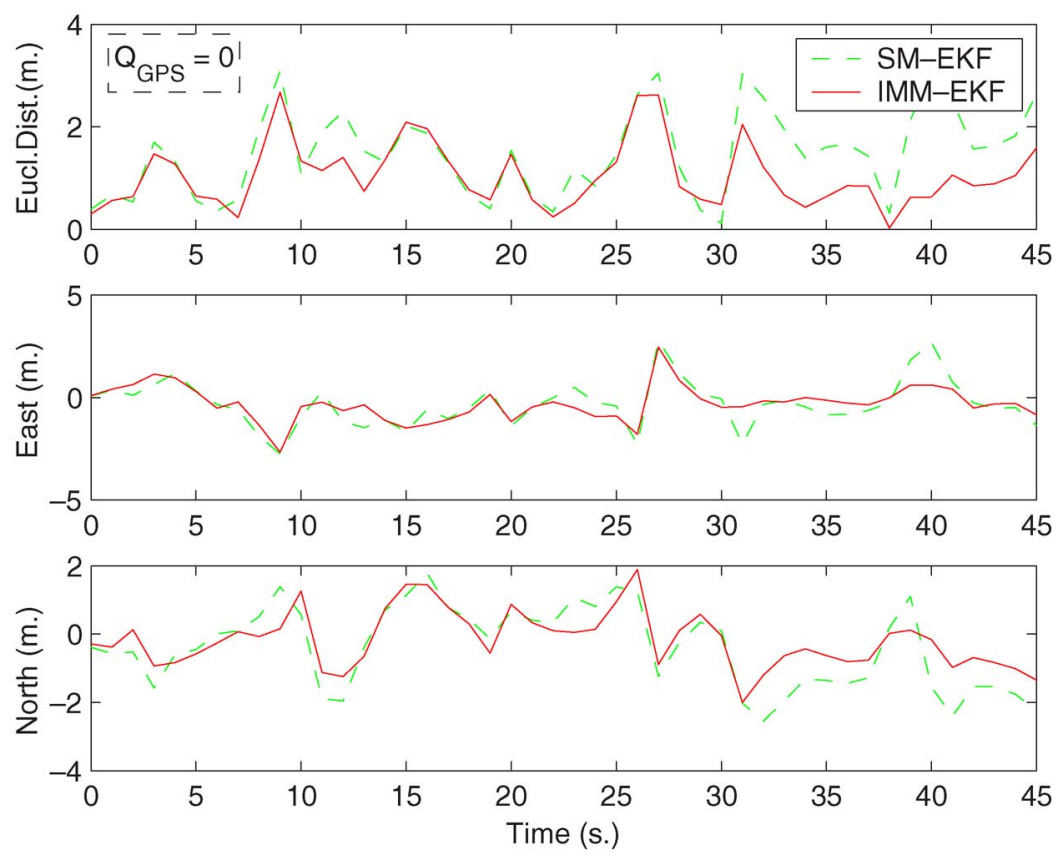

Fig. 19. Comparison of the position error estimate obtained by SM-EKF and IMM-EKF in scenario 10.

TABLE VIII

GPS OUTAGES AND RMS VALUES FOR POSITION ESTIMATE ERRORS IN SCENARIO 10

\begin{tabular}{|c|c|c|}
\hline Filter & SM-EKF & IMM-EKF \\
\hline RMS & $1.703(\mathrm{~m})$. & $1.219(\mathrm{~m})$. \\
\hline GPS outage & No GPS & No GPS \\
\hline
\end{tabular}

referred to the DGPS reference. On the other hand, it is not possible to calculate real values for the HPL $\mathrm{SBAS}_{\mathrm{S}}$ parameter. Future trials will be done in order to compare the HTL and HPL $_{\text {SBAS }}$ solutions in real environments. Thus, in this paper, we will focus our attention on the goodness of the HTL parameter as an integrity parameter for a multisensor navigation system.

Fig. 21 shows the position quality $(Q)$, the ED of the solution (referred to the DGPS reference), and the HTL parameter during this test. In the upper graph, $Q=2$ indicates EGNOS quality, $Q=1$ indicates single GPS, and $Q=0$ indicates no GPS position. The HTL parameter, as described in Section II-F, will be our integrity indicator, which supplies continuous fully meaningful integrity information to the user.

In the lower image of Fig. 21, the HTL value (dash-dot line) presents significant dependence on the GPS coverage and the filter rejections for avoiding multipath errors. It also manifests how the reliability on the position decreases due to the integration process performed by the filter. When the geostationary satellite is visible, the HTL values are close to $6 \mathrm{~m}$, but higher values are logged when there is no GEO visibility (and SISNeT is not used).

The HTL value has been compared with the ED of the estimated position errors during trajectory (solid line). As can be seen, the estimated position errors also depend on satellite visibility while keeping good error values during gaps. The rms value for position estimate error in this trajectory was $3.55 \mathrm{~m}$ with $29 \mathrm{~s}$ of GPS outage.
Similarities between HTL and ED behaviors are also visible in this figure, while the HTL appears to be a safe estimator (6-sigma) of system position integrity.

According to the trust levels on the positioning logged during the whole trajectory in test scenario 11, the system performance may be considered as correct, with the HTL value remaining below $7 \mathrm{~m}$ most of the time, despite the frequent GPS and EGNOS outages.

\section{CONCLUSION}

A high-integrity navigation system suitable for mass-market location-based road applications has been presented.

In order to design a low-cost navigation onboard system that fulfills the requirements regarding continuity, accuracy, and provision of integrity information to the user, the possibilities offered by satellite navigation have been studied. Different tests performed by using single GPS, GPS/GLONASS, and GPS/EGNOS sensors have been analyzed in this paper. According to our investigations, the use of double constellation sensors has limited improvements as compared with the single GPS solution. However, further investigations must be done before rejecting a double constellation solution. In fact, according to our tests, the use of the European SBAS system EGNOS enriches the single GPS solution with more accuracy and useful integrity information. On the other hand, SISNeT, which provides EGNOS with messages through the Internet, offers broad availability of these messages, even in situations with low visibility of the geostationary satellite.

Despite the aforementioned improvements achieved by SBAS systems, the GPS/GLONASS/SBAS solution cannot fulfill current road application requirements, especially regarding the continuity of the solution in unfriendly environments such as built-up areas or tunnels, and some assistance positioning system is highly recommended. In this paper, low-cost 


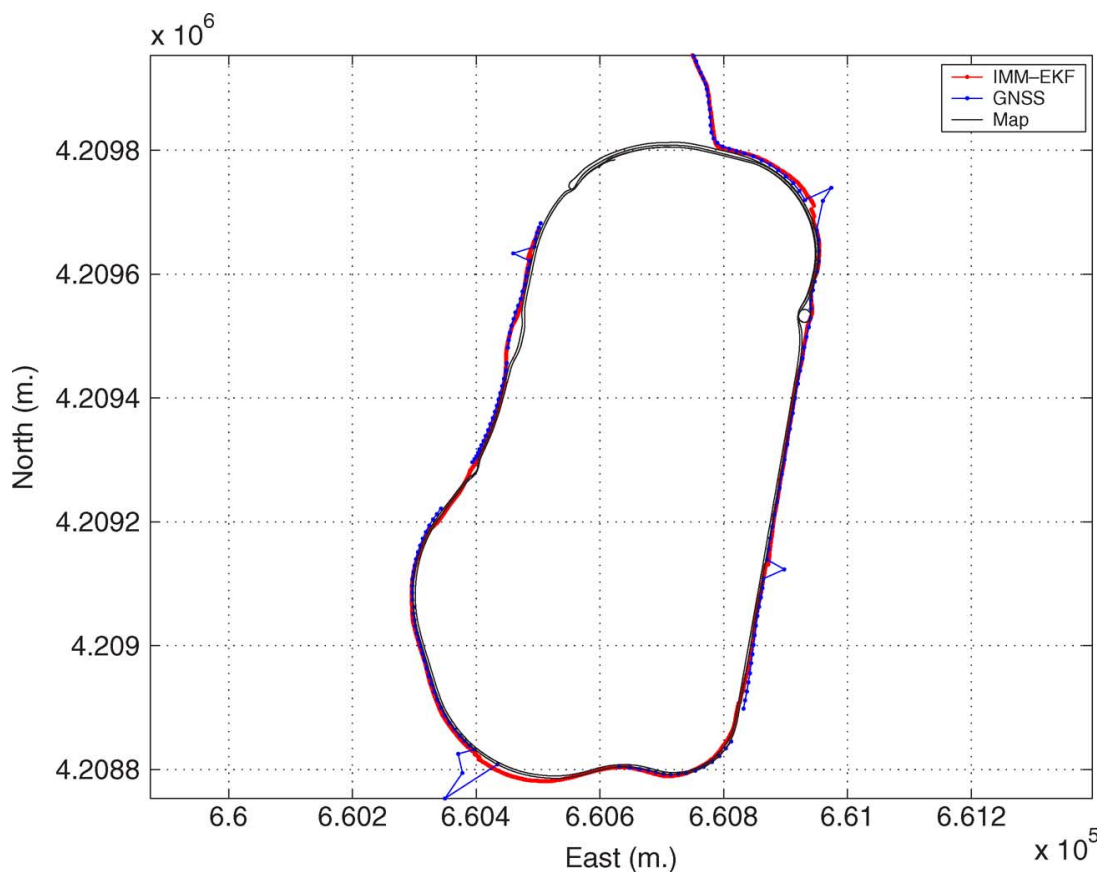

Fig. 20. Estimated vehicle trajectories in test scenario 11 for testing the HTL parameter.
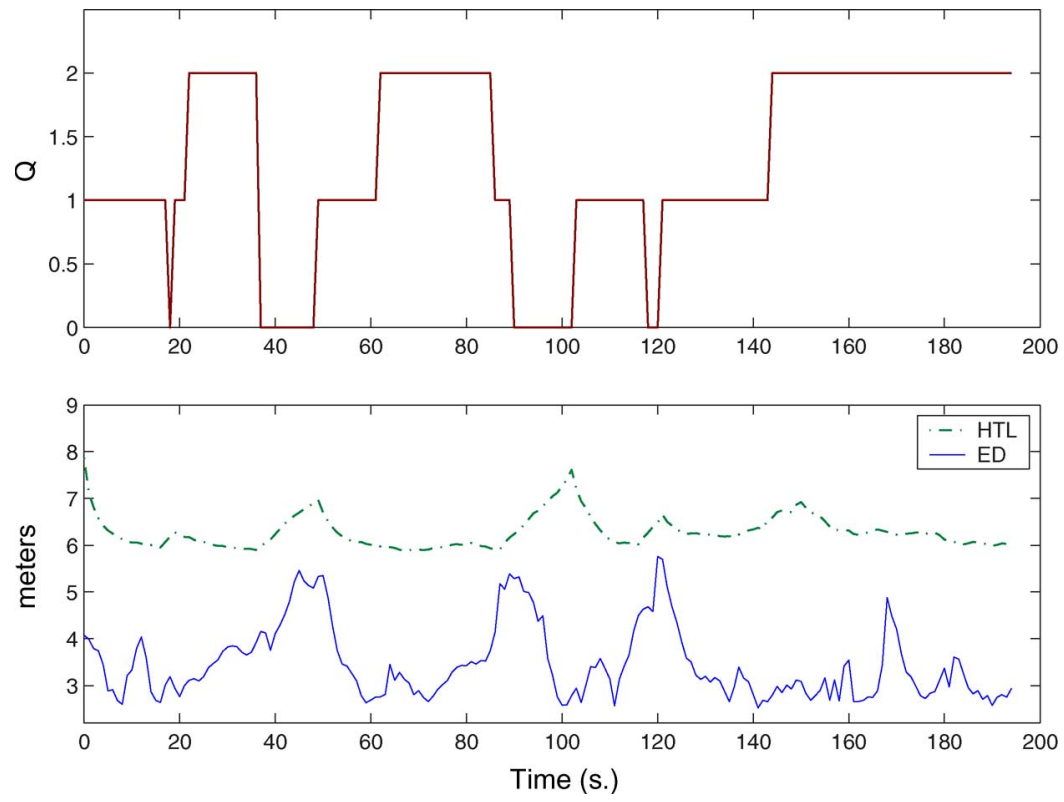

Fig. 21. Coverage and HTL values during the trajectory of test scenario 11.

inertial sensors based on MEM technology have proven to be useful in guaranteeing fault detection and continuous highly accurate positioning. Due to the bad noise features of these sensors and the accumulation of position error as a consequence of the double integration process required to obtain position from the acceleration measurements, error models were applied in order to diminish drifts in the absence of satellite coverage.

Regarding the filter, the implementation of a loosely coupled EKF architecture running an IMM method to combine two models of the vehicle allows the use of highly dynamic models, thus avoiding the increase of unrealistic noise considerations during nonmaneuvering situations. A classi- cal validation algorithm based on the Nyquist inequation has been implemented, and tests performed in real scenarios show the filter capability to detect and reject false measurements coming from the sensors. The proposed EKF has been compared to the UKF, where we obtained very similar results. The EKF option was finally chosen for the final IMM-based method for being most widely used in the literature.

With regard to the provision of integrity information to the user, the HTL parameter computed in our filter supplies real-time meaningful integrity information without the absence of the GNSS-based parameters, such as signal outages and uncomplete definition of the multisensor system accuracy. 
Future investigations will focus on the benefits of the IMMEKF method in localization and maneuvering recognition, considering a higher set of models and situations, and its influence on the HTL value.

\section{REFERENCES}

[1] P. A. Boysen and H. Zunker, "Low cost sensor hybridisation and accuracy estimation for road applications," in Proc. ESA Conf. Navitec, Noordwijk, The Netherlands, Dec. 2004.

[2] C. Boucher, A. Lahrech, and J. C. Noyer, "Non-linear filtering for land vehicle navigation with GPS outage," in Proc. IEEE Int. Conf. Syst., Man Cybern., The Hague, The Netherlands, Oct. 2004, pp. 1321-1325.

[3] C. Hay, Turn, Turn, Turn. Wheel-Speed Dead Reckoning for Vehicle Navigation. Santa Ana, CA: GPS World, Oct. 2005, pp. 37-42.

[4] B. Liu, M. Adams, and J. Ibañez-Guzman, "Multi-aided inertial navigation for ground vehicles in outdoor uneven environments," in Proc. IEEE Int. Conf. Robot. Autom., Barcelona, Spain, Apr. 2005, pp. 4703-4708.

[5] S. Rezaei and R. Sengupta, "Kalman filter based integration of DGPS and vehicle sensors for localization," in Proc. IEEE Int. Conf. Mechatronics Autom., Niagara Falls, ON, Canada, Aug. 2005, pp. 455-460.

[6] P. A. Boysen and H. Zunker, Integryty Hits the Road, Low Cost, High Trust for Mobile Units. Santa Ana, CA: GPS World, Jul. 2005, pp. 30-36.

[7] J. Wang and C. Wilson, Safety at the Wheel. Improving KGPS/INS Performance and Reliability. Santa Ana, CA: GPS World, May 2003, pp. 16-26.

[8] D. Berdjag and D. Pomorski, "DGPS/INS data fusion for land navigation," in Proc. Fusion, Stockholm, Sweden, Jun. 2004, pp. 881-887.

[9] A. Lahrech, C. Boucher, and J. C. Noyer, "Fusion of GPS and odometer measurements for map-based vehicle navigation," in Proc. IEEE Int. Conf. Ind. Technol., Hammamet, Tunisia, Dec. 2004, pp. 944-948.

[10] B. P. Zhang, J. Gu, E. Milios, and P. Huynh, "Navigation with IMU/GPS/digital compass with unscented kalman filter," in Proc. IEEE Int. Conf. Mechatronics Autom., Niagara Falls, ON, Canada, Jul. 2005, pp. 1497-1502.

[11] R. Lucas, "ESA projects on EGNOS applications," in Proc. Workshop EGNOS Performance Appl., Gdynia, Poland, Oct. 2005.

[12] N. Barbour and G. Schmidt, "Inertial sensor technology trends," IEEE Sensors J., vol. 1, no. 4, pp. 332-339, Dec. 2001.

[13] S. Sukkarieh, "Low cost, high integrity, aided inertial navigation systems for autonomous land vehicles," Ph.D. dissertation, Univ. Sydney, Sydney, Australia, 2000

[14] M. St-Pierre and D. Gingras, "Comparison between the unscented Kalman filter and the extended Kalman filter for the position estimation module of an integrated navigation information system," in Proc. IEEE Intell. Veh. Symp., Parma, Italy, Jun. 2004, pp. 831-835.

[15] K. Kobayashi, F. Munekata, and K. Watanabe, "A accurate navigation via sensor fusion of differential GPS and rate-gyro," in Proc. IEEE Int. Conf. Multisensor Fusion Integr. Intell. Syst., Las Vegas, NV, 1994, pp. 9-16.

[16] K. Kobayashi, F. Munekata, and K. Watanabe, "A low-cost GPS/Inertial attitude heading reference system (AHRS) for general aviation applications," in Proc. IMTC, Hamamatsu, Japan, May 1994, pp. 556-559.

[17] H. J. Hardt, D. Wolf, and R. Husson, "The dead reckoning localization system of the wheeled mobile robot ROMANE," in Proc. IEEE/SICE/RSJ Int. Conf. Multisensor Fusion Integr. Intell. Syst., 1996, pp. 603-610.

[18] S. Blackman and R. Popoli, Design and Analysis of Modern Tracking Systems. Norwood, MA: Artech House, 1999.

[19] Y. Bar-Shalom and W. Dale Blair, Multitarget-Multisensor Tracking: Applications and Advances, vol. III. Norwood, MA: Artech House, 2000.

[20] N. Kaempchen, K. Weiss, M. Schaefer, and K. C. J. Dietmayer, "IMM object tracking for high dynamic driving maneuvers," in Proc. IEEE Intell. Veh. Symp., Parma, Italy, Jun. 2004, pp. 825-830.

[21] L. Zhao and C. Thorpe, "Qualitative and quantitative car tracking from a range image sequence," in Proc. IEEE Comput. Soc. Conf. Comput. Vis. and Pattern Recog., 1998, pp. 496-501.

[22] K. Weiss, N. Kaempchen, and A. Kirchner, "Multiple-model tracking for the detection of lane change maneuvers," in Proc. IEEE Intell. Veh. Symp., Parma, Italy, Jun. 2004, pp. 937-942.

[23] A. F. Skarmeta, B. Úbeda, R. Toledo, and P. Tena, "Deployment Aspects and its Viability Scope Spanish Infrastructures," in Proc. Workshop German Model Toll Collection, Murcia, Spain, 2005.

[24] E. D. Kaplan, Understanding GPS. Principles and Applications. Norwood, MA: Artech House, 1996.
[25] RTCA DO-229C, Minimum Operational Performance Standards for Global Positioning System/Wide Area Augmentation System Airborne Equipment, Nov. 2001, Washington, DC: RTCA, Inc.

[26] Y. Bar-Shalom and X.-R. Li, Estimation and Tracking: Principles, Techniques and Software. Storrs, CT: YBS, 1998.

[27] Y. Bar-Shalom and X.-R. Li, Estimation and Tracking: Principles, Techniques and Software. Norwood, MA: Artech House, 1993.

[28] Y. K. Tham, H. Wang, and E. K. Teoh, "Adaptative state estimation for 4-wheel steerable industrial vehicles," in Proc. 37th IEEE Conf. Decision Control, Tampa, FL, 1998, pp. 4509-4514.

[29] B. Barshan and H. F. Durrant-Whyte, "Inertial navigation systems for mobile robots," IEEE Trans. Robot. Autom., vol. 11, no. 3, pp. 328-342, Jun. 1995.

[30] R. Toledo, "A high integrity navigation system for vehicles in unfriendly environments," Ph.D. dissertation, Univ. Murcia, Murcia, Spain, Nov. 2005.

[31] The Mathworks. [Online]. Available: http://www.mathworks.com/ products/optimization/description $3 . \mathrm{html}$

[32] J. J. Laviola, "A comparison of unscented and extended Kalman filtering for estimating quaternion motion," in Proc. Amer. Control Conf., Jun. 2003, pp. 2435-2440.

[33] S. Julier and J. K. Uhlmann, "Data fusion in nonlinear systems," in Handbook of Multisensor Data Fusion, D. Hall and J. Llinas, Eds. Boca Raton, FL: CRC, 2001

[34] A. N. Cui, L. Hong, and J. R. Layne, "A comparison of nonlinear filtering approaches with an application to ground target tracking," Signal Process., vol. 85, no. 8, pp. 1469-1492, Aug. 2005.

[35] S. Julier and J. K. Uhlmann, "A new extension of the Kalman filter to nonlinear systems," in Proc. AeroSense: 11th Int. Symp. Aerospace/Defence Sens., Simul. Controls, 1997, pp. 182-193.

[36] R. Toledo, M. A. Zamora, B. Úbeda, and A. F. Gómez-Skarmeta, "An integrity navigation system based on GNSS/INS for remote services implementation in terrestrial vehicles," in Proc. IEEE Intell. Transp. Syst. Conf., Washington, DC, 2004, pp. 477-480.

[37] J. Guivant, E. Nebot, and S. Baiker, "Autonomous navigation and map building using laser range sensors in outdoor applications," J. Robot. Syst., vol. 17 , no. 10 , pp. 565-583, 2000.

[38] S. Sukkarieh, E. Nebot, and H. Durrant-Whyte, "A high integrity IMU/GPS navigation loop for autonomous land vehicle applications," IEEE Trans. Robot. Autom., vol. 15, no. 3, pp. 572-578, Jun. 1999.

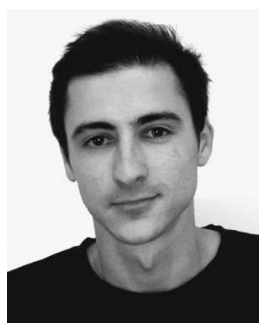

Rafael Toledo-Moreo received the M.S. degree in automation and electronics engineering from the Technical University of Cartagena (UPCT), Cartagena, Spain, in 2002 and the Ph.D. degree in computer science from the University of Murcia (UMU), Murcia, Spain, in 2006.

He is currently with the Department of Electronics and Computer Technology, UPCT, while he also collaborates with UMU in a number of projects concerning navigation systems applied to terrestrial vehicles. His main fields of interest are navigation systems, satellite and inertial navigation, and data fusion in road vehicle applications.

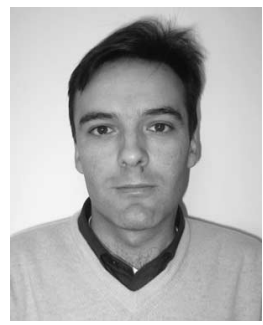

Miguel A. Zamora-Izquierdo received the M.S. degree in automation and electronics and the Ph.D. degree in computer science from the University of Murcia (UMU), Murcia, Spain, in 1997 and 2003, respectively.

In 1997, he was with the Department of Engineering and Systems, Technical University of Valencia (UPV), Valencia, Spain. Since 1999, he has been an Associate Professor with the Department of Information and Communication Engineering, UMU, where he works on several projects related to the ITS field and is especially focused on navigation systems for road vehicles. 


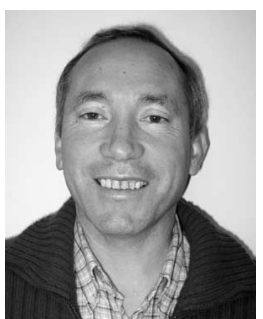

Benito Úbeda-Miñarro received the Bachelor's degree in telecommunication engineering and the Master's degree from the Technical University of Madrid, Madrid, Spain, in 1984 and 1992, respectively, and the $\mathrm{Ph} . \mathrm{D}$. degree in computer science from the University of Murcia, Murcia, Spain in 2006.

In 1987, he was with the Department of Signal Theory and Communications, UPM, where he was an Associate Professor. Since 1998, he has been with the Department of Computer Science, University of Murcia, where he is currently an Associate Professor. His main fields of interest are communications systems, global navigation satellite systems, and their applications in the ITS field.

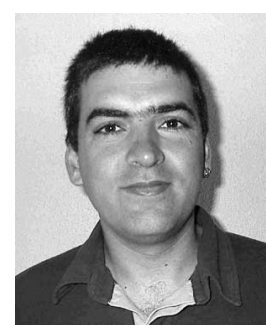

Antonio F. Gómez-Skarmeta received the M.S. degree in computer science from the University of Granada, Granada, Spain, and the B.S. (Hons.) and $\mathrm{Ph} . \mathrm{D}$. degrees in computer science from the University of Murcia, Murcia, Spain.

Since 1993, he has been a Professor with the Department of Information and Communication Engineering, University of Murcia. He has published more than 50 international papers. He has worked on different research projects in the national environment in the area of ITS. His main interests are in advanced services deployment in the area of mobility and ITS. 